\title{
The Equivalence of Ensembles for Classical Systems of Particles
}

\author{
Hans-Otto Georgii ${ }^{1}$
}

Received October 14, 1994

\begin{abstract}
For systems of particles in classical phase space with standard Hamiltonian, we consider (spatially averaged) microcanonical Gibbs distributions in finite boxes. We show that infinite-volume limits along suitable subsequences exist and are grand canonical Gibbs measures. On the way, we establish a variational formula for the thermodynamic entropy density, as well as a variational characterization of grand canonical Gibbs measures.
\end{abstract}

KEY WORDS: Classical statistical mechanics; microcanonical ensemble; variational principle; entropy; pressure.

\section{INTRODUCTION}

The equivalence of Gibbs ensembles is one of the central problems of statistical mechanics. As far as the thermodynamic functions are concerned, this question is already well understood. For example, it is well known that, under suitable conditions on the interaction, the infinite-volume limits of the entropy per volume and of the Gibbs free energy per volume exist and are related to each other by a Legendre-Fenchel transform; see, for example, refs. 3-5. A much deeper question is the equivalence of ensembles on the level of measures, which (in a possible but not optimal formulation) would mean that the microcanonical and the grand canonical Gibbs distributions in finite boxes have the same infinite-volume limits. In this paper we prove a version of the last statement which is not affected by the possibility of phase transitions.

We consider the standard setting of classical statistical mechanics. A particle in Euclidean space of dimension $d \geqslant 1$ is characterized by a pair $(x, p)$, where $x \in \mathbf{R}^{d}$ is the position and $p \in \mathrm{R}^{d}$ the momentum. A particle

\footnotetext{
${ }^{1}$ Mathematisches Institut der Universität Munich, Germany.
} 
configuration (without multiple occupancies) is thus given by a set $\omega \subset \mathbf{R}^{d} \times \mathbf{R}^{d}$ of the form

$$
\omega=\left\{\left(x, p_{x}\right): x \in \hat{\omega}\right\}
$$

where $\hat{\omega}$, the set of occupied positions, is a locally finite subset of $\mathrm{R}^{d}$, and $p_{x} \in \mathrm{R}^{d}$ is the momentum of the particle at position $x$. The set of all such configurations $\omega$ is denoted by $\Omega$. (In particular, $\Omega$ contains the empty configuration $\varnothing$.) We assume that the formal Hamiltonian has the standard form

$$
\begin{aligned}
H(\omega) & \equiv H^{\mathrm{kin}}(\omega)+H^{\mathrm{pot}}(\omega) \\
& =\sum_{x \in \hat{\omega}}\left|p_{x}\right|^{2}+\frac{1}{2} \sum_{x, y \in \hat{\omega}, x \neq y} \varphi(x-y)
\end{aligned}
$$

where $\varphi$ is a pair potential satisfying suitable stability conditions. (For convenience we assume throughout that the particle mass is equal to $1 / 2$.)

We are interested in the behavior of microcanonical Gibbs distributions in finite boxes in the infinite-volume limit. For simplicity we only consider the half-open cubes $\Lambda_{n}=\left[-n-1 / 2, n+1 / 2\left[{ }^{d}\right.\right.$ of volume $v_{n}=$ $(2 n+1)^{d}, n \geqslant 0$, which will often be considered as tori by assuming periodic boundary conditions. We write $M_{n \mid N, E \text {, per }}$ for the microcanonical Gibbs distribution for $N$ particles in $\Lambda_{n}$ with the energy constraint $H_{n \text {, per }} \leqslant E$, where $H_{n, \text { per }}$ is the Hamiltonian in $A_{n}$ with periodic boundary condition; see (2.5). We also consider the microcanonical Gibbs distributions $M_{n \mid N, E, \text { per }}^{u}$ on the energy shells $\left\{E-u<H_{n, \text { per }} \leqslant E\right\}$ of thickness $u>0$, and the microcanonical distributions $M_{n \mid N, E \text {, per }}^{0}$ on the energy surfaces $\left\{H_{n, \text { per }}=E\right\}$; see (2.13), (2.15), and lemma 6.3 for precise definitions. We shall also deal with other than periodic boundary conditions.

Here is an outline of our results. Under fairly general hypotheses on the pair interaction $\varphi$ we will show that, in the limit as $n \rightarrow \infty$ and $N / v_{n} \rightarrow \rho, E / v_{n} \rightarrow \varepsilon$ for an admissible pair $(\rho, \varepsilon)$ of particle and energy densities, the sequence $\left(M_{n \mid N, E \text {. per }}\right)_{n \geqslant 0}$ is relatively (sequentially) compact in a fairly strong topology, and each accumulation point belongs to the class $\mathscr{M}_{\rho, \varepsilon}$ of all translation-invariant probability measures $P$ on $\Omega$ which have expected particle density $\rho$ and mean energy $\varepsilon$ and maximize the mean entropy under this constraint (Theorem 3.3). Clearly, $\mathscr{M}_{\rho, \varepsilon}$ is contained in $\mathscr{G}_{z, \beta}$, the set of all $P$ that minimize the mean free energy for a suitably chosen activity $z>0$ and inverse temperature $\beta>0$. Our second main result is a variational principle stating that $\mathscr{G}_{z, \beta}$ consists of all tempered, translation-invariant (grand canonical) Gibbs measures for $z, \beta$ (Theorem 3.4). These two results together imply that every accumulation point of the 
microcanonical distributions $M_{n \mid N, E \text {, per }}$ is a Gibbs measure (in the grand canonical sense) for suitable parameters $z, \beta>0$. This proves the equivalence of ensembles on the level of measures in a formulation which still makes sense in the presence of phase transitions. If there is no phase transition, i.e., if $\mathscr{G}_{z, \beta}$ contains a unique element $P$, then

$$
M_{n \mid N, E, \text { per }} \rightarrow P
$$

in the above-mentioned topology. In fact, this convergence already holds when $P$ is the unique tempered Gibbs measure with expected particle and energy densities $(\rho, \varepsilon)$. A main step in the derivation of the preceding results will be to show that the thermodynamic entropy density can be characterized by a variational formula involving the mean entropy of translation-invariant probability measures on $\Omega$ (Theorem 3.2). As a matter of fact, it is the variational characterization of thermodynamic quantities in terms of states which will enable us to lift the equivalence of ensembles from the level of thermodynamic functions to the level of states.

The same results will be obtained for microcanonical distributions on thick or thin energy shells, as well as for free or configurational boundary conditions under an additional spatial averaging. As is easy to see from the proofs, our results can also be extended to the case when the kinetic energy is given by any positive power of the momenta. They also remain true for pure positional ensembles of particles without momentum, at least as long as no microcanonical distributions on energy shells are considered.

Our proofs make essential use of some ideas from large-deviation theory and rely, in particular, on the developments in refs. 1 and 2. These papers will be referred to as I and II, respectively. For example, Theorem II.1 stands for Theorem 1 of ref. 2 .

In Section 2 we introduce our conditions on the pair interaction $\varphi$ and describe the general setup. The main results are stated in Section 3. In Section 4 we investigate the mean entropy of translation-invariant states, and in Section 5 we derive the variational characterization of the thermodynamic entropy density. The subject of Section 6 is the asymptotics of microcanonical distributions, whereas the final Section 7 is devoted to the variational principle for Gibbs measures.

We conclude this introduction with a few bibliographic notes. A classical approach to the equivalence of ensembles (first proposed by Khinchin ${ }^{(6)}$ is to use a local central limit theorem for the particle number and energy. This was carried out in refs. 7-10 both for continuous systems and for lattice systems. The drawback of this method is that it works only in situations where a good local limit theorem is available-which certainly requires, at least, the absence of phase transitions. For classical lattice 
systems, Martin-Löf ${ }^{(1)}$ developed a "thermodynamic" approach to the equivalence of ensembles. It works also in the presence of phase transitions and makes essential use of translation invariance and variational principles, as we do here. A quite different approach is to define microcanonical, resp. canonical, Gibbs states directly in infinite volume in analogy to the familiar procedure in the grand canonical case, and to show that these are convex mixtures of the usual grand canonical Gibbs measures with different parameters. This idea was pursued in refs. $12-17$ both in the lattice and the continuous case. It is again not affected by the presenceof phase transitions, but the underlying concept of equivalence-though natural in the context of infinite-volume time evolutions-is not the traditional concept which is the subject of the present paper.

\section{THE SETTING}

\subsection{The Interaction}

We start with our assumptions on the pair interaction $\varphi$, an even measurable function from $\mathrm{R}^{d}$ to $\mathrm{R} \cup\{\propto\}$.

(A1) $\varphi$ is regular. That is, there exists a decreasing function $\psi:[0, \infty[\rightarrow[0, \infty[$ and a number $r(\varphi)<\infty$ such that

$$
\begin{array}{ll}
\varphi(x) \geqslant-\psi(|x|) & \text { for all } \quad x \in \mathrm{R}^{d} \\
\varphi(x) \leqslant \psi(|x|) & \text { whenever } \quad|x| \geqslant r(\varphi)
\end{array}
$$

and

$$
\int_{0}^{\infty} \psi(s) s^{d-1} d s<\infty
$$

(A2) Either $\varphi=\infty$ on a neighborhood of the origin (hard-core case), or $\varphi<\infty$ Lebesgue-almost everywhere, and $\varphi$ is non-integrably divergent at the origin, in that there exists a decreasing function $\chi:] 0, \infty[\rightarrow[0, \infty[$ such that

$$
\varphi(x) \geqslant \chi(|x|) \quad \text { when }|x| \text { is small enough }
$$

and

$$
\int_{0}^{1} \chi(s) s^{d-1} d s=\infty
$$


As is well known, ${ }^{(3)}$ (A1) and (A2) together imply that $\varphi$ is superstable. By definition, this means that there exist constants $a>0, b<\infty$ such that for all $n$

$$
H_{n} \geqslant a T_{n}-b N_{n}
$$

In the above, $H_{n} \equiv H_{n, \text { free }}$, the Hamiltonian in the box $A_{n}$ with free boundary condition, is given by $H_{n}(\omega)=H\left(\omega_{n}\right)$, where $H$ is as in (1.1) and $\omega_{n}=\omega \cap\left(A_{n} \times \mathrm{R}^{d}\right)$ is the restriction of $\omega \in \Omega$ to $A_{n} ; N_{n}(\omega)=\operatorname{card}\left(\hat{\omega}_{n}\right)$ is the particle number in $A_{n}$; and

$$
T_{n}=\sum_{i \in A_{n} \cup Z^{d}} N_{C+i}^{2}
$$

is the sum of the squared particle numbers $N_{C+i}(\omega)=\operatorname{card}(\hat{\omega} \cap(C+i))$ in the disjoint unit cells $C+i$ in $\Lambda_{n}$, where $C=A_{0}=\left[-1 / 2,1 / 2\left[{ }^{d}\right.\right.$ stands for the centered unit cube.

The superstability and regularity of $\varphi$ will be sufficient as long as we deal only with free or periodic boundary conditions. The latter lead to the periodic Hamiltonians

$$
\begin{aligned}
H_{n, \mathrm{per}}(\omega) & =\sum_{x \in \omega_{n}}\left|p_{x}\right|^{2}+\frac{1}{2} \sum_{x \in \omega_{n}, y \in \omega^{(n)}, y \neq x} \varphi(y-x) \\
& =H_{n}(\omega)+\frac{1}{2} \sum_{x \in \omega_{n}, y \in \omega^{(n)} \backslash A_{n}} \varphi(y-x)
\end{aligned}
$$

Here $\omega^{(n)}=\left\{(x+(2 n+1) i, p):(x, p) \in \omega_{n}, i \in Z^{d}\right\}$ is the periodic continuation of $\omega_{n}$.

The nonintegrable divergence of $\varphi$ at the origin will become important as soon as configurational boundary conditions are involved. For $t>0$ we define

$$
\Omega(t)=\left\{T_{n} / v_{n} \leqslant t \text { for all } n \geqslant 0\right\}
$$

The configurations in $\Omega^{*}=\bigcup_{t>0} \Omega(t)$ are called tempered. If $\varphi$ even has a hard core we use the same symbols $\Omega(t)$ and $\Omega^{*}$ to denote the set of all admissible hard-core configurations. (This avoids a messy distinction of cases.) For each $\zeta \in \Omega^{*}$ and $n \geqslant 0$ we let

$$
H_{n, \zeta}(\omega)=H_{n}(\omega)+\sum_{x \in \dot{\omega}_{n}, y \in \backslash A_{n}} \varphi(y-x)
$$

denote the Hamiltonian in $A_{n}$ with tempered boundary condition $\zeta$. The regularity of $\varphi$ ensures that the last sum exists; cf. Lemma II.4.2. 


\subsection{Translation-Invariant States}

Next we consider the class of possible states of our particle system. The configuration space $\Omega$ is equipped with the $\sigma$-algebra $\mathscr{F}$ which is generated by the counting variables $N(B): \omega \rightarrow \operatorname{card}(\omega \cap B)$ for Borel sets $B \subset \mathrm{R}^{d} \times \mathrm{R}^{d}$. It is well known ${ }^{(18)}$ that $\mathscr{F}$ is the Borel $\sigma$-algebra for the Polish topology on $\Omega$ that is induced by the mappings $N(h)$ : $\omega \rightarrow \sum_{x \in \omega} h\left(x, p_{x}\right), h: \mathbf{R}^{d} \times \mathbf{R}^{d} \rightarrow \mathrm{R}$ continuous with compact support.

We consider the class $\mathscr{P}$ of all probability measures ("states") $P$ on $(\Omega, \mathscr{F})$ satisfying

$$
\int P(d \omega) \sum_{x \in \omega_{n}}\left(1+\left|p_{x}\right|^{2}\right)<\infty \quad \text { for all } n
$$

as well as the class $\mathscr{P}_{\theta}$ of all $P \in \mathscr{P}$ which are invariant under the translation group $\Theta=\left(\vartheta_{x}\right)_{x \in \mathbb{R}^{d}}$ acting on $\Omega$ via $\vartheta_{x} \omega=\{(y-x, p):(y, p) \in \omega\}$. The mapping $(x, \omega) \rightarrow \vartheta_{x} \omega$ is known ${ }^{(18)}$ to be measurable.

For each $P \in \mathscr{P}_{\Theta}$ there exists a number $\rho(P)<\infty$, the intensity or mean particle number of $P$, such that $P\left(N_{A}\right)=\rho(P)|\Lambda|$ for all measurable $\Lambda \subset \mathrm{R}^{d}$; here we write $N_{A}=N\left(\Lambda \times \mathrm{R}^{d}\right)$ for the counting variable associated to $\Lambda,|\Lambda|$ is the Lebesgue measure of $A$, and $P(f)=\int f d P$ stands for the integral of a function $f$ with respect to $P$.

Also, for $P \in \mathscr{P}_{\theta}$ there exists ${ }^{(18,1)}$ a unique finite measure $P^{\circ}$ on $\mathrm{R}^{d} \times \Omega$, the Palm measure of $P$, such that

$$
\int P(d \omega) \sum_{x \in \omega} F\left(x, p_{x}, \vartheta_{x} \omega\right)=\int d x \int P^{\circ}(d p, d \omega) F(x, p, \omega)
$$

for all measurable functions $F \geqslant 0$ on $\mathrm{R}^{d} \times \mathrm{R}^{d} \times \Omega$. In particular, $P^{\circ}$ is supported on the set $\{(p, \omega):(0, p) \in \omega\}$, and the first marginal $\mu_{P}=P^{\circ}(\cdot \times \Omega)$ of $P^{\circ}$ satisfies

$$
\int P(d \omega) \sum_{x \in \omega_{n}} f\left(p_{x}\right)=v_{n} \int f d \mu_{P}
$$

for all $n \geqslant 0$ and measurable $f: \mathrm{R}^{d} \rightarrow\left[0, \infty\left[\right.\right.$. We call $\mu_{P}$ the momentum intensity measure.

We introduce a topology $\tau_{\mathscr{L}}$ on $\mathscr{P}$ (which is much finer than the weak topology associated to the above-mentioned Polish topology on $\Omega$ ) as follows. Let $\mathscr{L}$ denote the class of all measurable functions $f: \Omega \rightarrow \mathbf{R}$ which are local and tame in the sense that $f(\omega)=f\left(\omega_{l}\right)$ and

$$
|f(\omega)| \leqslant c\left(1+\sum_{x \in \omega_{t}}\left(1+\left|p_{x}\right|\right)\right) \equiv c\left(1+\tilde{N}_{l}(\omega)\right)
$$


for some $l \geqslant 0, c<\infty$, and all $\omega \in \Omega$. The topology $\tau_{\mathscr{L}}$ of local convergence is then defined as the weak* topology on $\mathscr{P}$ relative to $\mathscr{L}$, i.e., as the smallest topology making the mappings $P \rightarrow P(f) \equiv \int f d P$ with $f \in \mathscr{L}$ continuous. In particular, the mean particle density $\rho(P)$ and the mean momentum per particle $\int p \mu_{P}(d p) / \rho(P)$ are continuous functions of $P \in \mathscr{P}_{\boldsymbol{\theta}}$.

\subsection{The Gibbs ensembles}

For a fixed box $A_{n}$ and particle number $N$ we consider the set

$$
\Omega_{n \mid N}=\left\{\omega \in \Omega: \hat{\omega} \subset A_{n}, N_{n}(\omega)=N\right\}
$$

of all $N$-particle configurations in $\Lambda_{n}$, and the associated "Lebesgue measure" (or "Liouville measure")

$$
L_{n \mid N}(A)=\frac{1}{N !} \int_{A_{n}^{N}} d x_{1} \cdots d x_{N} \int_{\mathrm{R}^{d N}} d p_{1} \cdots d p_{N} 1_{A}\left(\left\{\left(x_{1}, p_{1}\right), \ldots,\left(x_{N}, p_{N}\right)\right\}\right)
$$

$A \in \mathscr{F} .\left(L_{n \mid 0}\right.$ gives weight 1 to the unique element $\varnothing$ of $\Omega_{n \mid 0}$.) The microcanonical distributions are obtained by conditioning $L_{n \mid N}$ on an energy shell of the form $\left\{E-u<H_{n, \mathrm{bc}} \leqslant E\right\}$ for some $E \in \mathrm{R}, 0<u \leqslant \infty$, and a boundary condition bc $\in \Omega^{*} \cup\{$ per $\}$. That is, wet set

$$
M_{n \mid N, E, \mathrm{bc}}^{u}(d \omega)=1_{\left\{E-u<H_{n, \mathrm{bc}} \leqslant E\right\}}(\omega) L_{n \mid N}(d \omega) / Z_{n \mid N, E, \mathrm{bc}}^{u}
$$

whenever the parameters are chosen in such a way that the normalizing constant

$$
Z_{n \mid N, E, \mathrm{bc}}^{u}=L_{n \mid N}\left(E-u<H_{n, \mathrm{bc}} \leqslant E\right)
$$

is positive. [(2.3) implies that it is always finite.] In the case $u=\infty$ (which means that there is no lower bound on the energy) we shall omit the upper index $u$. We call $Z_{n \mid N, E \text {, bc }}$ the microcanonical partition function or the phase volume.

We will also consider microcanonical distributions on thin energy shells $\left\{H_{n, \mathrm{bc}}=E\right\}$. Formally, these are defined by

$$
M_{n \mid N, E, \mathrm{bc}}^{0}=\lim _{u \rightarrow 0} M_{n \mid N, E . \mathrm{bc}}^{u}
$$

It is well known that these limits exist in the vague sense when $H_{n, \text { bc }}$ is a $C^{2}$ function [which certainly holds if $\varphi$ is $C^{2}$ with derivatives satisfying an 
analog of (2.1)]; see, for example, Khinchin ${ }^{(6)}$ or Section 3.5 of Tjur. ${ }^{(19)}$ However, we do not need any smoothness of $\varphi$. Indeed, since we are only interested in the asymptotic behavior of $M_{n \mid N, E_{1} \text { bc }}^{0}$ as $n \rightarrow \infty$, we only need to define $M_{n \mid N, E \text {, bc }}^{0}$ for events in a microscopic subregion of $A_{n}$, and for such events the limit (2.15) exists without any additional assumption on $\varphi$ (due to the smoothing effect of the kinetic energy). We defer the details until Section 6.

Finally, for the nonperiodic boundary conditions $b c \in \Omega^{*}$, we will have to deal with the averaged microcanonical distributions

$$
\bar{M}_{n \mid N . E, \mathrm{bc}}^{u}=v_{n}^{-1} \int_{\Lambda_{n}} d x M_{n \mid N . E, \mathrm{bc}}^{u} \circ \vartheta_{x}^{-1}
$$

This spatial averaging guarantees the asymptotic translation invariance we need.

In the grand canonical ensemble, the parameters $N$ and $E$ are replaced by the positive parameters $z$, the activity, and $\beta$, the inverse temperature. The grand canonical Gibbs distribution in $\Lambda_{n}$ with boundary condition $b c \in \Omega^{*} \cup\{$ per $\}$ and parameters $z, \beta$ is defined by

$$
G_{n, z, \beta, \mathrm{bc}}(d \omega)=\Xi_{n, z, \beta, \mathrm{bc}}^{-1} z^{N_{n}(\omega)} \exp \left[-\beta H_{n, \mathrm{bc}}(\omega)\right] L_{n}(d \omega)
$$

where $L_{n}=\sum_{N \geqslant 0} L_{n \mid N}$ is the Lebesgue measure on $\Omega_{n}=\left\{\omega \in \Omega: \hat{\omega} \subset \Lambda_{n}\right\}$, and

$$
\Xi_{n, z, \beta, \mathrm{bc}}=L_{n}\left(z^{N_{n}} \exp \left[-\beta H_{n, \mathrm{bc}}\right]\right)
$$

is the grand canonical partition function (which is finite; cf. the estimates in the proof of Lemma 5.2).

A measure $P \in \mathscr{P}$ is called a tempered Gibbs measure for $z, \beta>0$ if $P\left(\Omega^{*}\right)=1$ and, for all $n \geqslant 0$ and measurable functions $f \geqslant 0$ on $\Omega$,

$$
P(f)=\int P(d \zeta) \int G_{n, z, \beta, \zeta}(d \omega) f\left(\omega_{n} \cup\left(\zeta \backslash \zeta_{n}\right)\right)
$$

Clearly, the form of our Hamiltonian implies that, relative to all $G_{n, z, \beta, \zeta}$ and thus all tempered Gibbs measures, the momenta of the particles are conditionally i.i.d. with a Maxwellian (i.e., normal) distribution when the set $\hat{\omega}$ of occupied positions is given. For functions $f$ that depend only on $\omega,(2.18)$ is equivalent to the equilibrium equations introduced by Ruelle. ${ }^{(20)}$ 


\section{RESULTS}

A basic ingredient of our results is the existence of the mean energy and mean entropy of any $P \in \mathscr{P}_{\theta}$. The mean energy is defined by

$$
U(P)=\lim _{n \rightarrow \infty} v_{n}^{-1} P\left(H_{n}\right)
$$

This limit exists in $R \cup\{\infty\}$. Indeed, (2.10) and (2.8) show that the kinetic contribution equals

$$
U^{\mathrm{kin}}(P)=\int|p|^{2} \mu_{P}(d p)=v_{n}^{-1} P\left(H_{n}^{\mathrm{kin}}\right)<\infty
$$

independently of $n$. On the other hand, it was shown in Theorem II.1 [under the assumptions (2.1) and (2.3)] that the mean potential energy

$$
U^{\mathrm{pot}}(P)=\lim _{n \rightarrow \infty} v_{n}^{-1} P\left(H_{n}^{\mathrm{pot}}\right)
$$

exists in $\left[a \rho(P)^{2}-b \rho(P), \infty\right]$ and satisfies

$$
U^{\mathrm{pot}}(P)=\int P^{\circ}(d p, d \omega) \frac{1}{2} \sum_{0 \neq x \in \infty} \varphi(x)
$$

if $P\left(N_{C}^{2}\right)<\infty$ and equals $+\infty$ otherwise. Moreover, (3.2), (3.4), and Theorem II.1 show that the functions $U^{\mathrm{kin}}$ and $U^{\mathrm{pot}}$, and thus also $U$, on $\mathscr{P}_{\Theta}$ are affine (even measure affine, in that they are affine with respect to convex mixtures formed by arbitrary probability measures on $\mathscr{P}_{\Theta}$ ) and lower semicontinuous relative to $\tau_{\mathscr{L}}$.

The entropy of a state $P \in \mathscr{P}$ in $A_{n}$ is defined by

$$
S_{n}(P)= \begin{cases}-P_{n}\left(\log f_{n}\right) & \text { if } P_{n} \ll L_{n} \text { with density } f_{n} \\ -\infty & \text { otherwise }\end{cases}
$$

Where $P_{n}=P\left(\left\{\omega \in \Omega: \omega_{n} \in \cdot\right\}\right)$ is the restriction of $P$ to $A_{n}$. The following proposition on the mean entropy of invariant states will be proved in Section 4.

Proposition 3.1. For each $P \in \mathscr{P}_{\theta}$, the mean entropy

$$
S(P)=\lim _{n \rightarrow \infty} v_{n}^{-1} S_{n}(P)
$$


exists in $R \cup\{\infty\}$ and is a measure affine, upper semicontinuous function of $P$. The "energy-bounded" superlevel sets

$$
\left\{P \in \mathscr{P}_{\Theta}: S(P) \geqslant-c, U(P) \leqslant \varepsilon\right\}
$$

of $S$ (with $c, \varepsilon \in \mathrm{R}$ ) are compact and sequentially compact in $\tau_{\mathscr{L}}$.

We emphasize that $S$ fails to be upper semicontinuous with respect to the coarser topology $\tau_{\mathscr{L}^{b}}$ that is associated to the class $\mathscr{L}^{b}$ of all bounded local functions; see Example 4.3.

Our first main result concerns the existence and variational characterization of the thermodynamic entropy density. To state it we define for $\rho \geqslant 0$

$$
\varepsilon_{\min }(\rho)=\inf \left\{U(P): P \in \mathscr{P}_{\Theta}, \rho(P)=\rho, S(P)>-\infty\right\}
$$

By $(2.3), \varepsilon_{\min }(\rho) \geqslant a \rho^{2}-b \rho$, and $\varepsilon_{\min }(\cdot)$ is clearly convex. Also, $\varepsilon_{\min }(\cdot)$ is finite and continuous on a maximal interval $\left[0, \rho_{\max }\left[\right.\right.$, where $\rho_{\max }=\infty$ except when $\varphi$ has a hard core. These facts are proved in Lemma II.7.1 (which extends without difficulties to the present setting of particles with momentum). We introduce the convex set

$$
\Sigma=\left\{(\rho, \varepsilon): 0<\rho<\rho_{\max }, \varepsilon>\varepsilon_{\min }(\rho)\right\}
$$

and the abbreviation $\log _{-} u=\min (0, \log u)$.

Theorem 3.2. (a) Let $(\rho, \varepsilon) \in \Sigma$ and $t>0$. If $n \rightarrow \infty$ and $N, E, u$, bc run through any sequences such that $N / v_{n} \rightarrow \rho, E / v_{n} \rightarrow \varepsilon, v_{n}^{-1} \log _{-} u \rightarrow 0$, and $b c \in \Omega(t) \cup\{$ per $\}$, the limit

$$
s(\rho, \varepsilon)=\lim v_{n}^{-1} \log Z_{n \mid N, E . \mathrm{bc}}^{u}
$$

exists and admits the variational characterization

$$
s(\rho, \varepsilon)=\sup \left\{S(P): P \in \mathscr{P}_{\theta}, \rho(P)=\rho, U(P) \leqslant \varepsilon\right\}
$$

(b) The function $s(\cdot, \cdot)$ on $[0, \infty[\times \mathbf{R}$ defined by (3.9) is concave and upper semicontinuous. The set $\Sigma$ is the interior of the effective domain $\{s(\cdot, \cdot)>-\infty\}, s(\rho, \cdot)$ is strictly increasing on $] \varepsilon_{\min }(\rho), \infty[$ for each $\rho$, and the condition $U(P) \leqslant \varepsilon$ in (3.9) can be replaced by $U(P)=\varepsilon$.

We note that the existence of the limit in (3.8) has been known for a long time, at least for $b c=$ free. ${ }^{(3,4)}$ So our main point is its variational characterization (3.9); it is the microcanonical counterpart of the more 
familiar variational formula for the pressure which appears in Proposition 7.1. For $(\rho, \varepsilon) \notin \Sigma$, we still have the relation

$$
\lim \sup v_{n}^{-1} \log Z_{n \mid N, E, \mathrm{bc}}^{u} \leqslant s(\rho, \varepsilon)
$$

as is easy to see from the proof of Theorem 3.2 in Section 5.

Theorem 3.2 is the essential step toward the following main result, which will be proved in Section 6 .

Theorem 3.3. Suppose that $(\rho, \varepsilon) \in \Sigma, t>0, N / v_{n} \rightarrow \rho$ and $E / v_{n} \rightarrow \varepsilon$ as $n \rightarrow \infty$, and $u \in[0, \infty]$ and $b c \in \Omega(t) \cup\{$ per $\}$ are allowed to vary with $n$. Then the sequences $\left(M_{n \mid N, E, \text { per }}^{u}\right)_{n \geqslant 0}$ and $\left(\bar{M}_{n \mid N, E, \text { bc }}^{u}\right)_{n \geqslant 0}$ are (welldefined for large $n$ and) relatively sequentially compact in the topology $\tau_{\mathscr{L}}$, and every accumulation point belongs to the set

$$
\mathscr{M}_{\rho, \varepsilon}=\left\{P \in \mathscr{P}_{\theta}: \rho(P)=\rho, U(P)=\varepsilon, S(P)=s(\rho, \varepsilon)\right\}
$$

Our final task is to identify the "microcanonical equilibrium states" in $\mathscr{M}_{\rho, \varepsilon}$ as grand canonical Gibbs measures. For $(\rho, \varepsilon) \in \Sigma$ let $\beta=\beta(\rho, \varepsilon)>0$ be the derivative of $s(\rho, \cdot)$ at $\varepsilon$. This derivative is known to exist; see Rechtmann and Penrose(21) or Remark 6.5 below. In fact, it will come out indirectly from our results that $s(\cdot, \cdot)$ is also differentiable with respect to $\rho$; see Remark 3.7 below. For the moment, it is sufficient to observe that by concavity we can find a number $z>0$ such that, for a suitable constant $p(z, \beta) \in \mathbf{R}$, the plane

$$
\left(\rho^{\prime}, \varepsilon^{\prime}\right) \rightarrow p(z, \beta)+\beta \varepsilon^{\prime}-\rho^{\prime} \log z
$$

is a tangent to $s(\cdot, \cdot)$ at $(\rho, \varepsilon)$. In other words, $-\log z$ is an arbitrary element of the interval between the right and left derivatives of $s(\cdot, \varepsilon)$ at $\rho$. It then follows that for all $P \in \mathscr{P}_{\theta}$ with $U(P)<\infty$

$$
S(P) \leqslant s(\rho(P), U(P)) \leqslant p(z, \beta)+\beta U(P)-\rho(P) \log z
$$

with equality when $P \in \mathscr{M}_{\rho, \varepsilon}$. Introducing the mean free energy

$$
F_{z, \beta}(P)=\beta U(P)-\rho(P) \log z-S(P)
$$

we find that $F_{z ; \beta}(P) \geqslant-p(z, \beta)$ with equality for $P \in \mathscr{M}_{\rho, \varepsilon}$. Since $\mathscr{M}_{\rho, \varepsilon} \neq \varnothing$, we conclude that

$$
p(z, \beta)=-\min \left\{F_{z, \beta}(P): P \in \mathscr{P}_{\Theta}\right\}
$$

which is ( $\beta$ times) the pressure (see Proposition 7.1), and

$$
\mathscr{M}_{\rho, \varepsilon} \subset \mathscr{G}_{z, \beta} \equiv\left\{P \in \mathscr{P}_{\Theta}: F_{z, \beta}(P)=-p(z, \beta)\right\}
$$


The following variational principle asserts that $\mathscr{G}_{z, \beta}$ coincides with the set of all translation-invariant tempered Gibbs measures. Together with Theorem 3.3 and (3.12) this completes the proof of the equivalence of ensembles.

Theorem 3.4. For all $z, \beta>0$, the set of all translation-invariant tempered Gibbs measures for $z, \beta$ [as defined around (2.18)] coincides with the set $\mathscr{G}_{z, \beta}$ on which the mean free energy $F_{z, \beta}$ attains its minimum, $-p(z, \beta)$.

This result (which is well known for lattice systems ${ }^{(22-24)}$ ) will be proved in Section 7.

Remark 3.5. The preceding results obviously imply that the set $\mathscr{G}_{z, \beta}$ of translation-invariant tempered Gibbs measures is nonempty, which was proved first by Ruelle ${ }^{(20)}$ and Dobrushin. ${ }^{(25)}$ In fact, this follows already from (the proof of) Proposition 3.1 together with Theorem 3.4: $F_{z, \beta}$ has compact sublevel sets $\left\{P \in \mathscr{P}_{\Theta}: F_{z, \beta}(P) \leqslant c\right\}, c \in \mathrm{R}$, and thus attains its minimum.

Remark 3.6. $\mathscr{G}_{z, \beta}$ also contains all accumulation points of the relatively compact sequence $\left(G_{n, z, \beta} \text { per }\right)_{n \geqslant 0}$. This can be proved in complete analogy to Theorem 3.3; compare Proposition II.7.4. Thus, in the absence of phase transition when $\mathscr{G}_{z, \beta}=\{P\}$ we have

$$
P=\lim M_{n \mid N, E, \text { per }}^{u}=\lim G_{n, z, \beta, \text { per }}
$$

in $\tau_{\mathscr{L}}$, and a similar statement holds for spatially averaged distributions with boundary conditions in $\Omega(t)$ for some $t$. This expresses the equivalence of ensembles in terms of finite-volume Gibbs distributions.

Remark 3.7. The sets $\mathscr{G}_{z, \beta}(z, \beta>0)$ are pairwise disjoint. For, suppose that $\mathscr{G}_{z_{1}, \beta_{1}} \cap \mathscr{G}_{z_{2}, \beta_{2}} \neq \varnothing$. Then by (2.18) for any $n$ there exists some $\zeta \in \Omega^{*}$ such that, for $L_{n}$-almost all $\omega$,

$$
\Xi_{n, z_{i}, \beta_{i}, \zeta}^{-1} z_{i}^{N_{n}(\omega)} \exp \left[-\beta_{i} H_{n, \zeta}(\omega)\right]
$$

is independent of $i \in\{1,2\}$. Using this in the two cases $\omega=\varnothing$ and card $\hat{\omega}=1$, we readily find that $\left(z_{1}, \beta_{1}\right)=\left(z_{2}, \beta_{2}\right)$. It follows that for each $(\rho, \varepsilon) \in \Sigma$ there is a unique pair $(z, \beta)$ such that $(3.12)$ holds. By the argument leading to (3.12), this means that there is only one tangent plane to $s(\cdot, \cdot)$ at $(\rho, \varepsilon)$. Hence $s(\cdot, \cdot)$ is differentiable on $\Sigma$. (I am indebted to a referee for this observation.) 


\section{THE MEAN ENTROPY OF INVARIANT STATES}

In this section we prove Proposition 3.1. It is convenient to replace the infinite reference measures $L_{n}$ in (3.5) by consistent probability measures, namely the (local restrictions of) Poisson point random fields. Let $\tau: \mathbf{R}^{d} \rightarrow \mathbf{R} \cup\{\infty\}$ be any function satisfying $c(\tau) \equiv \int e^{-\tau(p)} d p<\infty$ and $Q^{\tau}$ the Poisson point random field with intensity measure $\mu^{\mathrm{T}}(d x, d p)=$ $d x e^{-\tau(p)} d p$. That is, $Q^{\tau}$ is the unique measure in $\mathscr{P}_{\theta}$ relative to which the particle numbers $N\left(B_{1}\right), \ldots, N\left(B_{k}\right)$ in disjoint phase space regions $B_{1}, \ldots, B_{k} \subset \mathrm{R}^{d} \times \mathrm{R}^{d}$ are independent and Poisson distributed with parameters $\mu^{\tau}\left(B_{i}\right), 1 \leqslant i \leqslant k$. By definition, its restriction $Q_{n}^{\mathrm{r}}$ to $A_{n}$ has the Radon-Nikodym density

$$
q_{n}^{\mathrm{\tau}}(\omega)=\exp \left[-\sum_{x \in \omega_{n}} \tau\left(p_{x}\right)-v_{n} c(\tau)\right]
$$

relative to $L_{n}$.

For any $P \in \mathscr{P}_{\Theta}$ we consider the relative entropy

$$
I\left(P_{n} ; Q_{n}^{\tau}\right)= \begin{cases}P_{n}\left(\log h_{n}\right) & \text { if } P_{n} \ll Q_{n}^{\tau} \text { with density } h_{n} \\ \infty & \text { otherwise }\end{cases}
$$

Clearly, $P_{n} \ll Q_{n}^{\tau}$ with a density $h_{n}$ if and only if $P_{n} \ll L_{n}$ with density $f_{n}=h_{n} q_{n}^{\tau}$. Hence we conclude from (3.5) and (2.10) that

$$
\begin{aligned}
S_{n}(P) & =-I\left(P_{n} ; Q_{n}^{\tau}\right)-P\left(\log q_{n}^{\tau}\right) \\
& =-I\left(P_{n} ; Q_{n}^{\tau}\right)+v_{n} \mu_{P}(\tau)+v_{n} c(\tau)
\end{aligned}
$$

We will use the following fact.

Lemma 4.1. For all $P \in \mathscr{P}_{\theta}$, the mean relative entropy

$$
I_{\tau}(P)=\lim _{n \rightarrow \infty} v_{n}^{-1} I\left(P_{n} ; Q_{n}^{\mathrm{r}}\right)
$$

exists in $[0, \infty]$. The function $I_{\tau}$ on $\mathscr{P}_{\Theta}$ is measure affine, and its sublevel sets $\left\{P \in \mathscr{P}_{\theta}: I_{\tau}(P) \leqslant c\right\}, c \geqslant 0$, are compact and sequentially compact in the topology $\tau_{\mathscr{L}^{b}}$ that is induced by the class $\mathscr{L}^{b}$ of all bounded local functions. If $\tau$ is such that

$$
\int e^{c|p|-\pi(p)} d p<\infty
$$

for all $c>0$, then the last assertion also holds in the topology $\tau_{\mathscr{L}}$. 
Proof. $\Omega$ can be identified in an obvious manner with $\left(\Omega_{0}\right)^{z^{d}}$, where $\Omega_{0}$ is defined in the line below (2.17). The first two sentences thus follow from the analogous result for lattice models [cf. Theorems (15.12) and (15.20) of ref. 24]. The last assertion is proved in Proposition I.2.6.

We are particularly interested in the case when $\tau(p)=\beta|p|^{2}$ for some $\beta>0$. We then replace the index $\tau$ by $\beta$. In particular, $Q^{\beta}$ is the ideal gas with particle density $c(\beta)=(\pi / \beta)^{d / 2}$ and Maxwellian momenta of variance $1 / 2 \beta$, and (4.2) takes the form

$$
v_{n}^{-1} S_{n}(P)=-v_{n}^{-1} I\left(P_{n} ; Q_{n}^{\beta}\right)+\beta U^{\mathrm{kin}}(P)+c(\beta)
$$

Since $U^{\mathrm{kin}}$ is finite, we conclude from Lemma 4.1 that $v_{n}^{-1} S_{n}(P)$ converges to

$$
S(P)=-I_{\beta}(P)+\beta U^{\mathrm{kin}}(P)+c(\beta)
$$

which is a measure affine function of $P$.

It remains to establish the continuity properties of $S$. To this end, we note first that a bound on the mean energy implies a bound on the mean kinetic energy. Indeed, since

$$
U^{\text {pot }}(P) \geqslant a \rho(P)^{2}-b \rho(P) \geqslant-b^{2} / 4 a
$$

by (2.3) and (3.3), we have the implication

$$
U(P) \leqslant \varepsilon \Rightarrow U^{\mathrm{kin}}(P) \leqslant \tilde{\varepsilon} \equiv \varepsilon+b^{2} / 4 a
$$

The proof of Proposition 3.1 is therefore completed by the following lemma.

Lemma 4.2. The mean entropy $S$ is upper semicontinous (relative to $\tau_{\mathscr{P}}$ ), and the restricted superlevel sets $\left\{S \geqslant c, U^{\text {kin }} \leqslant \tilde{\varepsilon}\right\}, c, \tilde{\varepsilon} \in \mathrm{R}$, are compact and sequentially compact.

Proof. In view of (4.3), the set in question is contained in the set $\left\{I_{1} \leqslant \tilde{c}\right\}$ with $\tilde{c}=-c+\tilde{\varepsilon}+c(1)$, and the latter set is compact and sequentially compact by Lemma 4.1 . We thus only need to show that $\{S \geqslant c\}$ is closed, i.e., that $S$ is upper semicontinuous. Applying (4.2) to the function $\tau=|\cdot|$, we see that

$$
S(P)=-I_{|\cdot|}(P)+\mu_{P}(|\cdot|)+c(|\cdot|)
$$

for all $P$. But $P \rightarrow \mu_{P}(|\cdot|)$ is continuous by definition of $\tau_{\mathscr{L}}$, and Lemma 4.1 asserts that $I_{|\cdot|}$ is lower semicontinous relative to $\tau_{\mathscr{L}^{b}} \subset \tau_{\mathscr{L}}$. This gives the result. 
We complete this section with an example showing that $S$ fails to be upper semicontinuous relative to $\tau_{\mathscr{L}^{h}}$.

Example 4.3. For each $k \geqslant 1$ let $B_{k} \subset \mathrm{R}^{d}$ be a Borel set of volume $e^{k^{2}} / k$ which is disjoint from the unit cube $C$. Let $\tau$ and $\tau_{k}$ be such that $e^{-\tau}=1_{C}$ and $e^{-\tau_{k}}=1_{C}+e^{-k^{2}} 1_{B_{k}}$. Now, $q_{n}^{\tau_{k}}$ converges to $q_{n}^{\tau} L_{n}$-almost everywhere, and thus in $L^{1}\left(L_{n}\right)$. Hence $Q^{\tau_{k}}$ converges in $\tau_{\mathscr{L}^{b}}$ (but not in $\left.\tau_{\mathscr{L}}\right)$ to $Q^{\tau}$. By $(4.2)$,

$$
\begin{aligned}
S\left(Q^{\tau_{k}}\right) & =-\int \tau_{k}(p) e^{-\tau_{k}(p)} d p+c\left(\tau_{k}\right) \\
& =k+1+1 / k \rightarrow \infty
\end{aligned}
$$

as $k \rightarrow \infty$, but on the other hand $S\left(Q^{\tau}\right)=1$.

\section{THE THERMODYNAMIC ENTROPY DENSITY}

This section contains the proof of Theorem 3.2. To begin we consider the function $s(\rho, \varepsilon)$ on $[0, \infty[\times \mathrm{R}$ defined by (3.9) (with the convention $\sup \varnothing=-\infty)$.

Lemma 5.1. (a) The supremum in (3.9) is attained whenever it is not $-\infty$.

(b) $s(\cdot, \cdot)$ is concave and upper semicontinuous, and its energybounded superlevel sets $\left\{(\rho, \varepsilon): s(\varepsilon, \rho) \geqslant c_{1}, \varepsilon \leqslant c_{2}\right\}, c_{1}, c_{2} \in \mathbf{R}$, are compact.

(c) The set $\Sigma$ in (3.7) is the interior of $\{s(\cdot, \cdot)>-\infty\}$.

Proof. Assertion (a), the upper semicontinuity, and the compactness of the energy-bounded superlevel sets are immediate consequences of Proposition 3.1 and the continuity of $\rho(\cdot)$. The concavity is clear because $S, \rho(\cdot)$, and $U$ are affine. Assertion (c) follows straight from the definitions.

Our main task is the proof of the convergence (3.8) toward the limit $s(\rho, \varepsilon)$ defined by (3.9). We shall proceed in three stages. In the first stage (which relies on refs. 1 and 2) we shall deal with "fattened" partition functions for which the particle number may range in a whole interval. In the second and third stages we shall remove the fattening by controlling the dependence of $Z_{n \mid N, E \text {, bc }}$ on the parameters $N$ and $E$.

The first stage is based on techniques from large-deviation theory. A central object in this theory is the translation-invariant empirical field

$$
R_{n, \omega}=v_{n}^{-1} \int_{A_{n}} \delta_{\vartheta_{x} \omega^{(n)}} d x \in \mathscr{P}_{\Theta}
$$


of a configuration $\omega$ in $\Lambda_{n}$. Here $\omega^{(n)}$ is as in (2.5). We shall take advantage of the formula

$$
H_{n, \mathrm{per}}(\omega)=v_{n} U\left(R_{n, \omega}\right)
$$

which follows from (3.2), (3.4), and the explicit formula (I.2.6) for the Palm measure $R_{n, \omega}^{\circ}$ of $R_{n, \omega}$. The following lemma allows us to proceed from periodic to nonperiodic boundary conditions.

Lemma 5.2. (a) For any $\varepsilon<\infty$ and $\delta>0$ there exists an integer $k=k(\varepsilon, \delta)$ such that for all $n$

$$
\left\{H_{n} \leqslant \varepsilon v_{n}\right\} \cap \Omega_{n} \subset\left\{H_{n+k, \text { per }} \leqslant(\varepsilon+\delta) v_{n}\right\}
$$

(b) For given $\varepsilon<\infty$ and $\delta, t>0$ there exists some $n(\varepsilon, \delta, t)$ such that for all $\zeta \in \Omega(t)$ and $n \geqslant n(\varepsilon, \delta, t)$

$$
\left\{H_{n, \zeta} \leqslant \varepsilon v_{n}\right\} \subset\left\{H_{n} \leqslant(\varepsilon+\delta) v_{n}\right\}
$$

Proof. (a) If $H_{n} \leqslant \varepsilon v_{n}$, then, by (2.3),

$$
N_{n}^{2} / v_{n} \leqslant T_{n} \leqslant v_{n} \varepsilon / a+b N_{n} / a
$$

and thus $T_{n} \leqslant t v_{n}$ for some $t<\infty$ depending on $\varepsilon$ (and $a, b$ ). The result thus follows from Lemma II.4.3.

(b) Here we use the nonintegrability of $\varphi$ at 0 . By Lemma II.6.1 there exists an increasing function $h: Z_{+} \rightarrow\left[0, \infty\left[\right.\right.$ with $h(l) / l^{2} \rightarrow \infty$ as $l \rightarrow \infty$, and a number $b^{\prime}>0$ such that

$$
H_{n} \geqslant T_{n}^{h}-b^{\prime} N_{n}
$$

for all $n$, where

$$
T_{n}^{h}=\sum_{i \in A_{n} \cap Z^{d}} h\left(N_{C+i}\right)
$$

On the other hand, Lemma II.6.3 asserts that for given $\delta^{\prime}>0$ and $n$ larger than some $n_{0}\left(\delta^{\prime}, t\right)$

$$
H_{n, \zeta} \geqslant H_{n}-\delta^{\prime} T_{n}^{h}-\delta^{\prime} v_{n}
$$

for all $\zeta \in \Omega(t)$. Suppose now that $H_{n, \zeta} \leqslant \varepsilon v_{n}$. Then (5.3) and (5.4) (with $\delta^{\prime}=1 / 2$ ) together show that

$$
T_{n}^{h} \leqslant(2 \varepsilon+1) v_{n}+2 b^{\prime} N_{n}
$$


whenever $n \geqslant n_{0}(1 / 2, t)$. Choosing $q$ such that $h(l) \geqslant l^{2}$ for $l \geqslant q$, we see that

$$
N_{n}^{2} / v_{n} \leqslant T_{n} \leqslant T_{n}^{h}+q^{2} v_{n}
$$

Combining this with the previous inequality, we find that $T_{n}^{h} \leqslant s v_{n}$ for some $s=s(\varepsilon)<\infty$ and all $n \geqslant n_{0}(1 / 2, t)$. Using (5.4) again with $\delta^{\prime}=\delta /(s+1)$, we can conclude.

Here is the first stage in the proof of (3.8).

Proposition 5.3. Let $\varepsilon<\infty$ and $D \subset[0, \infty[$ be a nondegenerate interval such that $(\rho, \varepsilon) \in \Sigma$ for some $\rho \in D$. Let $t>0$ and consider, for each $n$,

$$
a_{n \mid D, \varepsilon, \mathrm{bc}} \equiv L_{n}\left(N_{n} \in v_{n} D, H_{n, \mathrm{bc}} \leqslant v_{n} \varepsilon\right)=\sum_{N \in v_{n} D} Z_{n \mid N, v_{n} \varepsilon, \mathrm{bc}}
$$

where bc $\in \Omega(t) \cup\{$ per $\}$ may depend on $n$. Then

$$
\lim _{n \rightarrow \infty} v_{n}^{-1} \log a_{n \mid D, \varepsilon, \text { bc }}=\sup _{\rho \in D} s(\rho, \varepsilon)
$$

Proof. 1. We first derive the upper bound. We start with the case $\mathrm{bc}=$ per. Let $\beta>0$ be arbitrary. Equation (4.1) with $\tau=\beta|\cdot|^{2},(5.2)$, and (4.4) yield

$$
\begin{aligned}
a_{n \mid D, \varepsilon, \mathrm{per}} & =Q_{n}^{\beta}\left(1 / q_{n}^{\beta} ; \rho\left(R_{n}\right) \in D, U\left(R_{n}\right) \leqslant \varepsilon\right) \\
& \leqslant \exp \left[v_{n} \beta \tilde{\varepsilon}+v_{n} c(\beta)\right] Q_{n}^{\beta}\left(R_{n} \in A\right)
\end{aligned}
$$

where $A=\left\{P \in \mathscr{P}_{\theta}: \rho(P) \in \bar{D}, U(P) \leqslant \varepsilon\right\}$ and $\bar{D}$ is the closure of $D$. Since $A$ is closed in $\tau_{\mathscr{L}}$, we can use Theorem I.3.1 (with $Q=Q^{P}$ ) to obtain

$$
\begin{aligned}
\lim \sup v_{n}^{-1} \log a_{n \mid D, \varepsilon, \text { per }} & \leqslant \beta \tilde{\varepsilon}+c(\beta)-\inf _{P \in A} I_{\beta}(P) \\
& =\beta \tilde{\varepsilon}+\sup _{P \in A}\left[S(P)-\beta U^{\mathrm{kin}}(P)\right] \\
& \leqslant \beta \tilde{\varepsilon}+\sup _{\rho \in D} s(\rho, \varepsilon)
\end{aligned}
$$

The second step comes from (4.3), and in the last step we used the positivity of $U^{\text {kin }}$ and (3.9) together with the fact that (in view of our assumption on $D$ and Lemma 5.1) the suprema of $s(\cdot, \varepsilon)$ over $D$ and $\bar{D}$ coincide. Letting $\beta \rightarrow 0$ yields the upper bound in the case $b c=$ per. 
Turning to the case $\mathrm{bc}=$ free, we let $D^{\prime}$ be any interval containing $D$ in its interior, $\delta>0$, and $k$ be chosen according to Lemma 5.2(a). Then

$$
a_{n \mid D, \varepsilon, \text { free }} \leqslant a_{n+k \mid D^{\prime}, \varepsilon+\delta, \text { per }}
$$

whenever $n$ is so large that $v_{n+k} / v_{n}$ is sufficiently close to 1 . On the other hand, Lemma 5.1(b) implies that

$$
\inf _{D^{\prime} \supset D, \delta>0} \sup _{\rho \in D^{\prime}} s(\rho, \varepsilon+\delta)=\sup _{\rho \in D} s(\rho, \varepsilon)
$$

The upper bound for $b c=$ free thus follows from that for $b c=$ per.

Finally, suppose $\mathrm{bc}=\zeta \in \Omega(t)$ for some $t>0$, and choose any $\delta>0$. Lemma 5.2(b) then shows that

$$
a_{n \mid D, \varepsilon, \zeta} \leqslant a_{n \mid D, \varepsilon+\delta, \text { free }}
$$

for sufficiently large $n$, and the desired upper bound follows from the previous case.

2. We now turn to the lower bound. Since $U^{\mathrm{kin}} \geqslant 0$, we obtain as above

$$
a_{n \mid D, \varepsilon, \operatorname{per}} \geqslant \exp \left[v_{n} c(\beta)\right] Q_{n}^{\beta}\left(R_{n} \in A^{\circ}\right)
$$

for any $\beta>0$. Here $A^{\circ}=\left\{\rho(\cdot) \in D^{\circ}, U<\varepsilon\right\}$ and $D^{\circ}$ is the interior of $D$. Note that $A^{\circ}$ fails to be open because $U$ is only lower semicontinuous. A direct application of Theorem I.3.1 is therefore impossible. We rather need to extend the lower bound in Lemma II.7.2 to the present case of particles with momentum. To see that such an extension is valid we only need to control the additional kinetic term in lemma II.5.1. (The $\Phi$ there is our $U^{\text {pot }}$.) Using the notation in the proof of this lemma (in particular $m=n+k$ for a fixed $k$ ), we can write

$$
U^{\mathrm{kin}}\left(P^{(n)}\right)=v_{m}^{-1} P^{(n)}\left(H_{m}^{\mathrm{kin}}\right)=v_{m}^{-2} \int_{A_{m}} \hat{P}^{(n)}\left(H_{m}^{\mathrm{kin}} \circ \vartheta_{x}\right) d x
$$

Since $H_{m}^{\mathrm{kin}}$ is a sum of single-particle terms and $\hat{P}^{(n)}$ is $A_{m}$-periodic, the integrand above does not depend on $x$. Hence

$$
\begin{aligned}
U^{\mathrm{kin}}\left(P^{(n)}\right) & =v_{m}^{-1} \hat{P}^{(n)}\left(H_{m}^{\mathrm{kin}}\right)=v_{m}^{-1} P_{n}\left(H_{n}^{\mathrm{kin}} \mid \Gamma_{q(n)}\right) \\
& \leqslant p_{n}^{-1} v_{m}^{-1} P_{n}\left(H_{n}^{\mathrm{kin}}\right)=\left(v_{n} / p_{n} v_{m}\right) U^{\mathrm{kin}}(P)
\end{aligned}
$$


and therefore $\lim \sup _{n \rightarrow \infty} U^{\mathrm{kin}}\left(P^{(n)}\right) \leqslant U^{\mathrm{kin}}(P)$. This is all that is needed to extend Lemmas II.5.1 and II.7.2 to the present case. The result is

$$
\begin{aligned}
\liminf _{n \rightarrow \infty} v_{n}^{-1} \log a_{n \mid D, \varepsilon, \text { per }} & \geqslant c(\beta)-\inf _{P \in A^{\circ}} I_{\beta}(P) \\
& =\sup _{P \in A^{\circ}}\left[S(P)-\beta U^{\mathrm{kin}}(P)\right] \\
& \geqslant \sup _{\rho \in D} s(\rho, \varepsilon)-\beta \tilde{\varepsilon}
\end{aligned}
$$

In the last step we used (4.4) and the fact that the suprema of $s(\cdot, \varepsilon)$ over $D^{\circ}$ and $D$ coincide. Letting $\beta \rightarrow 0$, we obtain the lower bound in the case $\mathrm{bc}=$ per. The other boundary conditions can be treated by the same argument, with one addition: for $b c=$ free we also have to use the last estimate in the proof of Proposition II.5.4, whereas in the case bc $=\zeta \in \Omega(t)$ we argue as in the lines leading to Eq. (II.6.6).

The second step in the proof of (3.8) consists in controlling the variation of the microcanonical partition functions $Z_{n \mid N . E . b c}$ with respect to $N$. We need to distinguish the two cases in assumption (A2).

Lemma 5.4. Suppose that $\varphi<\infty$ a.e. Then for any given numbers $v, t>0$ there exist constants $c, u>0$ and $n_{0}$ such that

$$
Z_{n \mid N, E, \mathrm{bc}} \geqslant c Z_{n \mid N-1, E-u, \mathrm{bc}}
$$

whenever $N \leqslant w_{n}, E \in \mathrm{R}$, bc $\in \Omega(t) \cup\{\operatorname{per}\}$, and $n \geqslant n_{0}$.

Proof. Let $\varphi_{+}=\max (\varphi, 0)$ be the positive part of $\varphi$. Since $\varphi<\infty$ a.e., we can find some $q>0$ such that

$$
\int \min \left(1, \varphi_{+}(x) / q\right) d x \leqslant 1 / 4 v
$$

Indeed, the integrand in the integral on the left side converges to $1_{\left\{\varphi_{+}=\infty\right\}}=0$ a.e. as $q \rightarrow \infty$, and the regularity assumption (Al) ensures that the dominated convergence theorem is applicable. Next, for each $n, N, \omega \in \Omega_{n \mid N-1}$ and $(x, p) \notin \omega$ we can write

$$
H_{n, \mathrm{bc}}(\omega \cup\{(x, p)\})=H_{n, \mathrm{bc}}(\omega)+|p|^{2}+\sum_{y \in \omega} \varphi(y-x)+h_{n, \mathrm{bc}}(x)
$$

where, for example,

$$
h_{n, \zeta}(x)=\sum_{y \in \hat{\zeta} \backslash M_{n}} \varphi(y-x)
$$


for $\zeta \in \Omega(t)$. Using (2.1) and the arguments in Lemma II.4.2, we see that there exists some $r \geqslant r(\varphi)$ such that $h_{n, \mathrm{bc}}(x) \leqslant 1$ whenever $x \in A_{n-r}$, bc $\in \Omega(t) \cup\{$ per $\}$, and, in the case bc $=$ per, $\omega \in \Omega_{n \mid N}$ with $N \leqslant v v_{n}$. Let $N_{0}$ be so large that $v_{n-r} \geqslant v_{n} / 2$ when $n \geqslant n_{0}$. Then we have for arbitrary $n \geqslant n_{0}$ and $\omega \in \Omega_{n \mid N-1}$

$$
\begin{aligned}
\left|\left\{x \in A_{n-r}: \sum_{y \in \omega} \varphi(y-x) \leqslant q\right\}\right| & \geqslant \int_{A_{n-r}}\left[1-\sum_{y \in \omega} \min \left(1, \varphi_{+}(y-x) / q\right)\right] d x \\
& \geqslant v_{n-r}-(N-1) / 4 v \geqslant v_{n} / 4
\end{aligned}
$$

Setting $u=q+2$, we thus obtain the final estimate

$$
\begin{aligned}
N Z_{n \mid N, E, \mathrm{bc}} \geqslant & \int L_{n \mid N-1}(d \omega) \int_{A_{n-\mathrm{r}}} d x \int d p \\
& \times 1\left\{H_{n, \mathrm{bc}}(\omega) \leqslant E-u, \sum_{y \in \hat{\omega}} \varphi(y-x) \leqslant q,|p|^{2} \leqslant 1\right\} \\
\geqslant & \left(c_{1} v_{n} / 4\right) Z_{n \mid N-1, E-u, \mathrm{bc}}
\end{aligned}
$$

where $c_{1}$ is the volume of the unit ball in $R^{d}$. The lemma thus follows with $c=c_{1} / 4 v$.

If $\varphi$ has a hard core, we have an estimate in the opposition direction.

Lemma 5.5. Suppose $\varphi=\infty$ on a neighborhood of 0 . For given $v>0$ and $\varepsilon<\infty$ there exist constants $c, u>0$ and $n_{0}$ such that

$$
Z_{n \mid N, E, b c} \geqslant c Z_{n \mid N+1, E-u, b c}
$$

for all $n \geqslant n_{0}, N \geqslant v v_{n}, E \leqslant \varepsilon v_{n}$, and bc $\in \Omega^{*} \cup\{$ per $\}$.

Proof. The hard-core property and the regularity (2.1) imply the existence of a number $u>0$ such that

$$
\sum_{y \in \infty} \varphi(y-x) \geqslant-u
$$

for all $x \in \mathrm{R}^{d}$ and all admissible hard-core configurations $\hat{\omega}$. This gives the estimate

$$
\begin{aligned}
(N+1) Z_{n \mid N+1, E-u, \mathrm{bc}} & \leqslant \int L_{n \mid N}(d \omega) \int_{\Lambda_{n}} d x \int d p 1_{\left\{H_{n, \mathrm{bc}}(\omega)+|p|^{2} \leqslant E\right\}} \\
& =v_{n} \int d p Z_{\left.n|N, E-| p\right|^{2}, \mathrm{bc}}
\end{aligned}
$$


In view of Lemma 5.7 below, the last expression is at most

$$
v_{n} \int d p e^{-r|p|^{2}} Z_{n \mid N, E . \mathrm{bc}}
$$

for some $\gamma=\gamma(\nu, \varepsilon)>0$, provided $N \geqslant v v_{n}, E \leqslant \varepsilon v_{n}$, and $n$ is large enough. The lemma is now obvious.

The next result marks the second stage in the proof of (3.8).

Proposition 5.6. Let $(\rho, \varepsilon) \in \Sigma$ and $t>0$. In the limit as $n \rightarrow \infty$, $N / v_{n} \rightarrow \rho, \quad E / v_{n} \rightarrow \varepsilon, \quad$ and $\quad \mathrm{bc}=\mathrm{bc}(n) \in \Omega(t) \cup\{\operatorname{per}\}, \quad v_{n}^{-1} \log Z_{n \mid N, E, \mathrm{bc}}$ converges to the function $s(\rho, \varepsilon)$ defined by (3.9).

Proof. If $D \subset[0, \infty$ [ is a nondegenerate interval containing $\rho$ in its interior and $\varepsilon^{\prime}>\varepsilon$, then $Z_{n \mid N, E \text {, bc }} \leqslant a_{n \mid D, \varepsilon^{\prime}, \text { bc }}$ eventually. Proposition 5.3 and the upper semicontinuity of $s(\cdot, \cdot)$ thus imply that

$$
\lim \sup v_{n}^{-1} \log Z_{n \mid N, E, \text { bc }} \leqslant s(\rho, \varepsilon)
$$

For the lower bound we consider first the case when $\varphi<\infty$ a.e. We fix any $v>\rho$ and let $u$ and $0<c<1$ be as in Lemma 5.4. Let $\delta>0$ be so small that $\delta<\rho$ and $\varepsilon-u \delta>\varepsilon_{\min }(\rho)$. For sufficiently large $n$ we have $N / v_{n} \leqslant v$ and, by $k$-fold iteration of Lemma 5.4 ,

$$
Z_{n \mid N, E, \mathrm{bc}} \geqslant c^{k} Z_{n \mid N-k, E-k u, \mathrm{bc}}
$$

Averaging the right-hand side over all $k$ between 0 and $\delta v_{n}$ and choosing any $\left.\varepsilon^{\prime} \in\right] \varepsilon_{\min }, \varepsilon-\delta[$ and a nondegenerate closed interval $D \subset] \rho-\delta, \rho[$, we obtain

$$
Z_{n \mid N, E, \mathrm{bc}} \geqslant c^{\delta v_{n}}\left(\delta v_{n}\right)^{-1} a_{n \mid D, \varepsilon^{\prime}, \mathrm{bc}}
$$

when $n$ is large enough. Proposition 5.3 thus gives

$$
\lim \inf v_{n}^{-1} \log Z_{n \mid N, E \text {, bc }} \geqslant \delta \log c+s\left(\rho^{\prime}, \varepsilon^{\prime}\right)
$$

for any $\rho^{\prime} \in D$. Letting $\rho^{\prime} \rightarrow \rho, \delta \rightarrow 0, \varepsilon^{\prime} \rightarrow \varepsilon$ in this order, we can conclude. In the case when $\varphi$ has a hard core we can proceed analogously, using Lemma 5.5 instead of Lemma 5.4.

The final step in the proof of (3.8) is the consideration of energy shells. To this end we need to control the $E$ dependence of the $Z_{n \mid N, E \text {, bc }}$. Let $\hat{L}_{n \mid N}$ be the "Lebesgue measure" on the set $\hat{\Omega}_{n \mid N}=\left\{\hat{\omega} \subset A_{n}\right.$ : card $\left.\hat{\omega}=N\right\}$ of 
position configurations of $N$ particles in $A_{n}$ (with the standard $\sigma$-algebra), that is,

$$
\int f d \hat{L}_{n \mid N}=\frac{1}{N !} \int_{\Lambda_{n}^{N}} d x_{1} \cdots d x_{N} f\left(\left\{x_{1}, \ldots, x_{N}\right\}\right)
$$

for measurable $f \geqslant 0$ on $\hat{\Omega}_{n \mid N}$. Note that $H_{n, \mathrm{bc}}^{\mathrm{pot}}$ can be considered as a function of $\hat{\omega}$.

Lemma 5.7. For all $n, N, E$, and bc we have

$$
Z_{n \mid N, E, \mathrm{bc}}=c_{N} \int \hat{L}_{n \mid N}(d \hat{\omega})\left(E-H_{n, \mathrm{bc}}^{\mathrm{pot}}(\hat{\omega})\right)_{+}^{N d / 2}
$$

where $c_{N}$ is the volume of the unit ball in $\mathrm{R}^{N d}$. In particular, $Z_{n \mid N .} E_{\text {, bc }}$ is differentiable with respect to $E$ with derivative

$$
Z_{n \mid N, E, \mathrm{bc}}^{\prime}=c_{N}(N d / 2) \hat{L}_{n \mid N}\left(\left(E-H_{n, \mathrm{bc}}^{\mathrm{pot}}\right)_{+}^{(N d / 2)-1}\right)
$$

which is sometimes called the structure function. ${ }^{(6)}$ Also, for every $v>0$ and $\varepsilon<\infty$ there exists a constant $\gamma=\gamma(v, \varepsilon, t)>0$ such that

$$
Z_{n \mid N, E-u, \mathrm{bc}} \leqslant e^{-\gamma u} Z_{n \mid N, E, \mathrm{bc}}
$$

whenever $N \geqslant v v_{n}, E \leqslant \varepsilon v_{n}, u \geqslant 0, \mathrm{bc} \in \Omega(t) \cup\{$ per $\}$ for a given $t>0$, and $n$ is large enough.

Proof. Equation (5.6) follows from (2.12) and (2.14) by integration over the momenta $p_{1}, \ldots, p_{N}$. Equation (5.7) is obtained by an interchange of differentiation and integration, which is justified by the dominated convergence theorem. Turning to the proof of (5.8), we fix any $t>0$. By the proof of Lemma 5.2(b) there exists a number $c>-\varepsilon$ (depending on $\varepsilon$ and $t)$ such that, for all bc $\in \Omega(t) \cup\{$ per $\}$ and sufficiently large $n, H_{n \text { bc }}^{\text {pot }} \geqslant-c v_{n}$ whenever $H_{n, \mathrm{bc}}^{\mathrm{pot}} \leqslant \varepsilon v_{n}$. Hence $\left(E-H_{n, \mathrm{bc}}^{\mathrm{pot}}\right)_{+} \leqslant(\varepsilon+c) v_{n}$ and therefore

$$
\frac{d}{d E} \log Z_{n \mid N . E, \mathrm{bc}}=Z_{n \mid N . E, \mathrm{bc}}^{\prime} / Z_{n \mid N, E, \mathrm{bc}} \geqslant \gamma \equiv \nu d / 2(\varepsilon+c)
$$

(5.8) now follows by integration.

It follows immediately from (5.8) and Proposition 5.6 that $s(\rho, \cdot)$ is strictly increasing on $] \varepsilon_{\min }(\rho), \infty[$. This in turn shows that the condition " $U(P) \leqslant \varepsilon$ " in (3.9) can be replaced by " $U(P)=\varepsilon$." Inequality (5.8) further implies that

$$
\left(1-e^{-v \bar{u}}\right) Z_{n \mid N, E, \mathrm{bc}} \leqslant Z_{n \mid N, E, \mathrm{bc}}^{u} \leqslant Z_{n \mid N, E, \mathrm{bc}}
$$


for a suitable $\gamma>0$ whenever $N / v_{n} \rightarrow \rho, E / v_{n} \rightarrow \varepsilon, u>0$, bc $\in \Omega(t) \cup\{$ per $\}$ for a given $t$, and $n$ is large enough. Combining this inequality with Proposition 5.6, we arrive at (3.8). The proof of Theorem 32 is therefore complete.

Remark 5.8. Under the conditions of (3.8) we also have that

$$
s(\rho, \varepsilon)=\lim v_{n}^{-1} \log Z_{n \mid N, E . \text { bc }}^{\prime}
$$

[cf. (5.7)]. This follows from (5.9) and the inequality

$$
Z_{n \mid N, E, \mathrm{bc}}^{\prime} \leqslant \int_{0}^{1} d u Z_{n \mid N, E+u, \mathrm{bc}}^{\prime}=Z_{n \mid N, E+1, \mathrm{bc}}^{1}
$$

\section{THE ASYMPTOTICS OF MICROCANONICAL DISTRIBUTIONS}

This section contains the proof of Theorem 3.3. Let $(\rho, \varepsilon) \in \Sigma$ and

$$
N / v_{n} \rightarrow \rho, \quad E / v_{n} \rightarrow \varepsilon, \quad \text { bc } \in \Omega(t) \cup\{\text { per }\}
$$

for some fixed $t>0$ as $n \rightarrow \infty$. In a first step, we shall prove Theorem 3.3 under the additional hypothesis

$$
v_{n}^{-1} \log _{-} u \rightarrow 0 \quad \text { as } n \rightarrow \infty
$$

which appears in Theorem 3.2. In this case we follow an idea devised in ref. 26. In a second step we then extend the result to the alternate case when $u$ vanishes or tends to 0 exponentially with $v_{n}$.

Suppose (6.1) and (6.2) hold. For brevity we write $M_{n}=M_{n \mid N, E, \text { bo }}^{u}$ and $\bar{M}_{n}=\bar{M}_{n \mid N, E, \text { bc }}^{u}$. Theorem 3.2 implies that these measures are well defined for sufficiently large $n$. Let $M_{n}^{\text {per }} \in \mathscr{P}$ denote the measure relative to which the configurations in the disjoint blocks $A_{n}+(2 n+1) i, i \in Z^{d}$, are independent with identical distribution $M_{n}$, and

$$
\tilde{M}_{n}=v_{n}^{-1} \int_{A_{n}} M_{n}^{\text {per }} \circ \vartheta_{x}^{-1} d x \in \mathscr{P}_{\Theta}
$$

the associated invariant average. We shall derive the asymptotics of $\bar{M}_{n}$ from that of $\tilde{M}_{n}$. First, we get from (3.2) that

$$
U^{\mathrm{kin}}\left(\tilde{M}_{n}\right)=v_{n}^{-2} \int_{A_{n}} M_{n}^{\mathrm{per}}\left(H_{n}^{\mathrm{kin}} \circ \vartheta_{x}\right) d x=v_{n}^{-1} M_{n}\left(H_{n}^{\mathrm{kin}}\right)
$$


The second equality follows from the periodicity of $M_{n}^{\text {per }}$ and the additive nature of $H_{n}^{\text {kin }}$. Together with Lemma 5.2(b) and a version of (4.4), this implies that

$$
\limsup _{n \rightarrow \infty} U^{\mathrm{kin}}\left(\tilde{M}_{n}\right)<\infty
$$

Next we consider the mean entropy of $\tilde{M}_{n}$. The following key estimate follows from Theorem 3.2.

Proposition 6.1. Under conditions (6.1) and (6.2),

$$
\lim _{\inf _{n \rightarrow \infty}} S\left(\tilde{M}_{n}\right) \geqslant s(\rho, \varepsilon) .
$$

Proof. Let $Q=Q^{1}$ and $I=I_{1}$ be as in (4.3) for $\beta=1$. Lemma I.5.5 asserts that $I\left(\tilde{M}_{n}\right) \leqslant v_{n}^{-1} I\left(M_{n} ; Q_{n}\right)$. Together with (4.3), (6.3), and (4.2) this yields

$$
\begin{aligned}
S\left(\tilde{M}_{n}\right) & \geqslant-v_{n}^{-1} I\left(M_{n} ; Q_{n}\right)+v_{n}^{-1} M_{n}\left(H_{n}^{\mathrm{kin}}\right)+c(1) \\
& =v_{n}^{-1} S_{n}\left(M_{n}\right)=v_{n}^{-1} \log Z_{n \mid N, E, \mathrm{bc}}^{\prime \prime}
\end{aligned}
$$

and Theorem 3.2 gives the result.

Let us say that two sequences $\left(P_{1, n}\right)$ and $\left(P_{2, n}\right)$ in $\mathscr{P}$ are asymptotically equivalent, and write $P_{1, n} \sim P_{2, n}$ as $n \rightarrow \infty$, if for all $f \in \mathscr{L}$

$$
\lim _{n \rightarrow \infty}\left(P_{1, n}(f)-P_{2, n}(f)\right)=0
$$

Considering again the empirical fields $R_{n}$ in (5.1), we define the measures $M_{n} R_{n+k}=\int M_{n}(d \omega) R_{n+k, \omega} \in \mathscr{P}_{\mathcal{O}}$.

Lemma 6.2. Assuming (6.1) and (6.2), we have for each $k \geqslant 0$

$$
\tilde{M}_{n} \sim M_{n} R_{n+k} \sim \bar{M}_{n} \quad \text { as } n \rightarrow \infty
$$

If $\mathrm{bc}=$ per, then, in addition, $\bar{M}_{n} \sim M_{n}$ as $n \rightarrow \infty$.

Proof. We only prove the first asymptotic equivalence. A similar but simpler argument shows that $M_{n} R_{n} \sim \bar{M}_{n}$, and for bc=per one has $M_{n} R_{n}(f)=M_{n}(f)$ when $f \in \mathscr{L}$ and $n$ is so large that $f$ only depends on $\omega_{n}$.

Equations (4.3) and (6.4) together with Proposition 6.1 imply that

$$
\limsup _{n \rightarrow \infty} I\left(\tilde{M}_{n}\right)<\infty
$$

where again $I=I_{1}$ is the mean relative entropy with respect to the Poisson point random field $Q=Q^{1}$. Lemma I.5.7 thus yields that $\tilde{M}_{n} \sim M_{n} R_{n}$ as 
$n \rightarrow \infty$, which is the case $k=0$. For general $k$, the argument in the proof of this lemma shows that for each $f \in \mathscr{L}$ and arbitrary $a>0$

$$
\left|v_{n+k}^{-1} v_{n} \tilde{M}_{n}(f)-M_{n} R_{n+k}(f)\right| \leqslant o(1)+c \tilde{M}_{n, k}\left(\tilde{N}_{l} ; \tilde{N}_{l} \geqslant a\right)
$$

Here $l$ and $c$ are such that $f$ depends only on $\omega$, and (2.11) holds (where $\tilde{N}_{l}$ was defined), and $\tilde{M}_{n, k} \in \mathscr{P}_{\theta}$ is the average of the measures $M_{n, k}^{\text {per }} \circ \vartheta_{x}^{-1}$ with $x \in \Lambda_{n+k}$, where $M_{n, k}^{\mathrm{per}}$ is the measure relative to which the configurations in the disjoint blocks $A_{n+k}+(2 n+2 k+1) i, i \in Z^{d}$, are i.i.d. with distribution $M_{n}$. (In particular, $\tilde{M}_{n, 0}=\tilde{M}_{n}$, and for $k \geqslant 1$ there are $M_{n, k}^{\text {per }}$-almost surely no particles in the corridors at the boundaries of the blocks.)

To complete the proof we therefore need to show that $a$ can be chosen in such a way that the last term in (6.6) becomes arbitrarily small, uniformly in $n$. By Lemma I.5.2, this would follow if we knew that (6.5) holds for the sequence $\left(\tilde{M}_{n, k}\right)_{n \geqslant 0}$ in place of $\left(\tilde{M}_{n}\right)_{n \geqslant 0}$. But this is indeed the case because

$$
d Q_{n} / d Q_{n+k}=1_{\left\{N_{n+k}-N_{k}=0\right\}} \exp \left[\left(v_{n+k}-v_{n}\right) c(1)\right]
$$

and therefore

$$
v_{n} I\left(\tilde{M}_{n, k}\right) \leqslant I\left(M_{n} ; Q_{n+k}\right)=I\left(M_{n} ; Q_{n}\right)+\left(v_{n+k}-v_{n}\right) c(1)
$$

Assertion (6.5) for the sequence $\left(\tilde{M}_{n, k}\right)_{n \geqslant 0}$ thus follows as in the case $k=0$.

We are now prepared for the first step in the proof of Theorem 3.3.

Proof of Theorem 3.3 Under Condition (6.2). By (6.4) and Lemmas 6.1 and 4.2 , the sequence $\left(\tilde{M}_{n}\right)_{n \geqslant 0}$ is sequentially compact, and every accumulation point $P$ satisfies $S(P) \geqslant s(\rho, \varepsilon)$. Since $\rho\left(\tilde{M}_{n}\right)=N / v_{n} \rightarrow \rho$ and $\rho(\cdot)$ is continuous, we have $\rho(P)=\rho$.

To show that $U(P) \leqslant \varepsilon$, we let $\mathscr{T}$ be an infinite set of positive integers such that $\bar{M}_{n} \rightarrow P$ as $n \rightarrow \infty$ through $\mathcal{A}$. We may assume that either $\mathrm{bc}=$ per for all $n \in . \mathcal{N}$ or bc $\in \Omega(t)$ for all $n \in \mathcal{A}$. For each $k$, Lemma 6.2 yields that $M_{n} R_{n+k} \rightarrow P$ as $n \rightarrow \infty$ through $\mathscr{H}$, and

$$
U\left(M_{n} R_{n+k}\right)=M_{n}\left(U\left(R_{n+k}\right)\right)=v_{n+k}^{-1} M_{n}\left(H_{n+k} \text { per }\right)
$$

because of (5.2). Since $M_{n}$ is supported on $\left\{H_{n, \text { bc }} \leqslant E\right\}$, for each $\delta>0$ we can find some $k$ such that $M_{n}\left(H_{n+k \text {, per }}\right) \leqslant(\varepsilon+\delta) v_{n}$ for sufficiently large $n \in \mathcal{N}$. Indeed, if $\mathrm{bc}=$ per we may simply take $k=0$, and in the case bc $\in \Omega(t)$ we can apply assertions (b) and (a) of Lemma 5.2. Since $U$ is lower semicontinuous, it follows that $U(P) \leqslant \varepsilon$. In fact, $U(P)=\varepsilon$ because 
otherwise $S(P)<s(\rho, \varepsilon)$ by the strict monotonicity of $s(\rho, \cdot)$. Hence $P \in \mathscr{M}_{\rho, \varepsilon}$.

By Lemma 6.2 , the above properties of the sequence $\left(\tilde{M}_{n}\right)$ carry over to the asymptotically equivalent sequence $\left(\bar{M}_{n}\right)$ and, in the case bc $=$ per, to $\left(M_{n}\right)$. This establishes Theorem 3.3 under assumption (6.2).

We now turn to the case when (6.2) fails, which means that $u$ tends to zero exponentially with $v_{n}$. Since this includes the case $u=0$, we first need a proper definition of the microcanonical distributions $M_{n \mid N . E \text {, be }}^{0}$ on the energy surfaces $\left\{H_{n, \mathrm{bc}}=E\right\}$. As we said in Section 2.3, it is sufficient to have a definition of $M_{n \mid N, E, \text { bc }}^{0}(f)$ when $f \in \mathscr{L}$ is fixed and $n$ is sufficiently large. Such a definition can be obtained in analogy to (5.7). We still assume (6.1).

Lemma 6.3. For each $m \geqslant 0$ there exists a number $n(m)$ such that, for all $n \geqslant n(m)$ and $0<u \leqslant 1, M_{n \mid N, E, \text { bc }}^{u}$ is well defined and the following holds: For any $f \in \mathscr{L}$ which only depends on the particle configuration in the union $A=\Lambda(f)$ of at most $m$ unit cells, the limit

$$
M_{n \mid N, E, \mathrm{bc}}^{0}(f)=\lim _{u \rightarrow 0} M_{n \mid N, E, \mathrm{bc}}^{u}(f)
$$

exists and equals $Z_{n \mid N, E, \mathrm{bc}}^{\prime}(f) / Z_{n \mid N, E, \mathrm{bc}}^{\prime}$, where

$$
\begin{aligned}
Z_{n \mid N, E, \mathrm{bc}}^{\prime}(f)= & \sum_{k \leqslant N-4 / d} \int L_{A \cap A, \mid k}(d \omega) f(\omega) \\
& \times \int \hat{L}_{A_{n} \backslash A \mid N-k}(d \hat{\eta}) b_{N-k, \omega, n, \mathrm{bc}}(E, \hat{\eta})
\end{aligned}
$$

In the above, the Lebesgue measures $L_{A \cap A_{n} \mid k}$ and $\hat{L}_{A_{n} \backslash A \mid N-k}$ are defined in obvious analogy to (2.12) and (5.5), and

$$
\begin{aligned}
& b_{N-k, \omega, n, \mathrm{bc}}(E, \hat{\eta}) \\
& \quad=c_{N-k}(N-k)(d / 2)\left(E-H^{\mathrm{kin}}(\omega)-H_{n, \mathrm{bc}}^{\mathrm{pot}}(\hat{\omega} \cup \hat{\eta})\right)_{+}^{(N-k) d / 2-1}
\end{aligned}
$$

with $c_{N-k}$ as in (5.6).

Proof. Note first that, by Remark 5.8, $Z_{n \mid N, E, \mathrm{bc}}^{u} \geqslant u Z_{n \mid N, E-u, \mathrm{bc}}^{\prime}>0$ when $0<u \leqslant 1$ and $n$ is sufficiently large. This means that for large $n$ the measures $M_{n \mid N, E \text {, be }}^{u}$ are well defined.

It follows directly from (6.8) that, for all $u>0$, the integral

$$
\int_{E-u}^{E} d s b_{N-k, \omega, n, \mathrm{bc}}(s, \hat{\eta})
$$


is the volume of the spherical shell consisting of all $\left(p_{1} \ldots, p_{N-k}\right) \in \mathrm{R}^{(N-k) d}$ such that

$$
\left.\left.\sum_{i=1}^{N-k}\left|p_{i}\right|^{2}+H^{\mathrm{kin}}(\omega)+H^{\mathrm{pot}}(\hat{\omega} \cup \hat{\eta}) \in\right] E-u, E\right]
$$

The $\hat{L}_{A_{n} \backslash A \mid N-k}(d \hat{\eta})$ integral of this volume equals

$$
\left.\left.L_{A_{n} \backslash A \mid N-k}\left(\eta: H_{n, \text { bc }}(\omega \cup \eta) \in\right] E-u, E\right]\right)
$$

Hence

$$
\int_{E-u}^{E} Z_{n \mid N, s, \mathrm{bc}}^{\prime}(f) d s=L_{n \mid N}\left(f ; E-u<H_{n, \mathrm{bc}} \leqslant E, N_{A} \leqslant N-4 / d\right)
$$

for all $u>0$. The condition $k \leqslant N-4 / d$ in (6.7) ensures that the exponent in (6.8) is positive, so that $Z_{n \mid N, s, b c}^{\prime}(f)$ is a continuous function of $s$. On the other hand, for large $n$ the condition $N_{A} \leqslant N-4 / d$ on the right-hand side of $(6.9)$ is vacuous because

$$
\left\{H_{n, \mathrm{bc}} \leqslant E\right\} \cap \Omega_{n \mid N} \subset\left\{N_{A} \leqslant N-4 / d\right\}
$$

when $n$ is large enough. To see this, we let $\omega \in \Omega_{n \mid N}$ and suppose that $N_{A}(\omega)>N-4 / d \equiv \underline{N}$. Then $T_{n}(\omega) \geqslant \underline{N}^{2} / m$ and therefore $H_{n}(\omega) \geqslant$ $a N^{2} / m-b N$. By an estimate similar to Lemma II.4.2, we find a constant $c>0$ such that

$$
H_{n, \mathrm{bc}}(\omega)-H_{n}(\omega) \geqslant\left\{\begin{array}{lll}
-c N t & \text { if } & \mathrm{bc} \in \Omega(t) \\
-c N^{2} / v_{n} & \text { if } & \mathrm{bc}=\mathrm{per}
\end{array}\right.
$$

Combining these estimates with (6.1), we see that $H_{n \text {, bc }}(\omega)>E$ when $n$ is large enough. The right-hand side of (6.9) is therefor equal to

$$
M_{n \mid N, E, \mathrm{bc}}^{u}(f) \int_{E-u}^{E} Z_{n \mid N, s, \mathrm{bc}}^{\prime} d s
$$

and the lemma follows from the continuity of the $Z^{\prime}$.

Our strategy for the extension of Theorem 3.3 to the case when (6.2) fails is a comparison of $M_{n \mid N, E, \text { bc }}^{u}$ with $M_{n \mid N, E, \text { bc }}^{s}$ for some $s \geqslant u$ satisfying $v_{n}^{-1} \log _{-} s \rightarrow 0$. This comparison is based on the monotonicity properties of $Z_{n \mid N, E, \text { be }}^{\prime}(f)$ and the next lemma.

Lemma 6.4. Suppose that $u \rightarrow 0$ as $n \rightarrow \infty$ and (6.1) holds. Then

$$
Z_{n \mid N, E+u, \mathrm{bc}}^{\prime} / Z_{n \mid N, E, \mathrm{bc}}^{\prime} \rightarrow 1 \text { as } n \rightarrow \infty
$$


Proof. The ratio under consideration is certainly not less than 1 . To obtain an upper estimate, we consider the function

$$
\sigma_{n}(s)=\log Z_{n \mid N, s, b c}^{\prime}
$$

for values of $n$ and $s$ for which $Z_{n \mid N, s, \text { bc }}^{\prime}>0$. Recall from (5.7) that, up to an additive constant, $\sigma_{n}(s)$ has the form $\log \hat{L}\left((s-\hat{H})_{+}^{\prime}\right)$, where we use the abbreviations $\hat{L}=\hat{L}_{n \mid N}, \hat{H}=H_{n \text {, bc }}^{\text {pot }}$, and $l=N d / 2-1$. Computing the derivatives as in Lemma 5.7, we obtain for each $s$

$$
\begin{aligned}
\left(\frac{1}{\sigma_{n}^{\prime}}\right)^{\prime}(s) & =1-\frac{l(l-1) \hat{L}\left((s-\hat{H})_{+}^{l-2}\right) \hat{L}\left((s-\hat{H})_{+}^{\prime}\right)}{l^{2} \hat{L}\left((s-\hat{H})_{+}^{l-1}\right)^{2}} \\
& \leqslant 1-\frac{l-1}{l}=\frac{2}{N d-2} \equiv a_{N}
\end{aligned}
$$

where the inequality follows from the Cauchy-Schwarz inequality. Hence

$$
1 / \sigma_{n}^{\prime}(s)-1 / \sigma_{n}^{\prime}(t) \leqslant a_{N}(s-t)
$$

when $s \geqslant t$, and the mean value theorem gives

$$
\delta v_{n} /\left[\sigma_{n}\left(E+\delta v_{n}\right)-\sigma_{n}(E)\right]-1 / \sigma_{n}^{\prime}(E) \leqslant \delta v_{n} a_{N}
$$

for each $\delta>0$. Letting $n \rightarrow \infty$ and $\delta \rightarrow 0$, we thus obtain from Remark 5.8

$$
\lim \sup \sigma_{n}^{\prime}(E) \leqslant \partial_{+} s(\rho, \varepsilon) / \partial \varepsilon
$$

The expression on the right side is the right derivative with respect to $\varepsilon$ (which exists by concavity). Averaging over $t$ in (6.10), we obtain

$$
1 / \sigma_{n}^{\prime}(E+u)-u /\left[\sigma_{n}(E+u)-\sigma_{n}(E)\right] \leqslant u a_{N}
$$

Together with (6.11) (for $E+u$ in place of $E$ ) this gives

$$
\lim \sup \left(\sigma_{n}(E+u)-\sigma_{n}(E)\right) \leqslant \lim \sup u \sigma_{n}^{\prime}(E+u)=0
$$

which is the desired estimate.

Remark 6.5. (6.12) also gives a counterpart to (6.11) which, together with the concavity of $s(\rho, \cdot)$, implies that $s(\rho, \cdot)$ is differentiable and

$$
\frac{\partial s(\rho, \varepsilon)}{\partial \varepsilon}=\lim \frac{d}{d E} \log Z_{n \mid N, E, \text { bc }}^{\prime}
$$

The same argument also shows that the last identity holds without the prime on the right-hand side. 
The following proposition asserts that microcanonical distributions on thin and on slightly thicker energy shells are asymptotically equivalent.

Proposition 6.6. Suppose (6.1) holds and $u, s \in[0, \infty]$ vary with $n$ in such a way that $0 \leqslant u \leqslant s \rightarrow 0$ and $v_{n}^{-1} \log s \rightarrow 0$. Then

$\bar{M}_{n \mid N, E, \mathrm{bc}}^{u} \sim \bar{M}_{n \mid N, E, \mathrm{bc}}^{s} \quad$ and $\quad M_{n \mid N, E, \mathrm{per}}^{u} \sim M_{n \mid N, E, \mathrm{per}}^{s}$ as $n \rightarrow \infty$

Proof. We drop all indices which are not necessary. We need to show that, for given $f \in \mathscr{L}, \quad \bar{M}_{n \mid \mathrm{bc}}^{u}(f)-\bar{M}_{n \mid \mathrm{bc}}^{s}(f) \rightarrow 0$ and $M_{n \mid \mathrm{per}}^{u}(f)-M_{n \mid \mathrm{per}}^{s}(f) \rightarrow 0$ as $n \rightarrow \infty$. We can assume without loss that $f \geqslant 0$. Let $l$ and $c$ be such that $f(\omega)=f\left(\omega_{l}\right)$ and $(2.11)$ holds, $n\left(v_{l+1}\right)$ be chosen according to Lemma 6.3, and $n \geqslant n\left(v_{t+1}\right)$. We define

$$
a_{n}(u, f) \equiv a_{n \mid E}(u, f)=\int_{0}^{1} Z_{n \mid E-r u}^{\prime}(f) d r
$$

and $a_{n}(u)=a_{n}(u, 1)$ for the constant function 1. Then $M_{n}^{u}(f)=$ $a_{n}(u, f) / a_{n}(u)$. [Note that $a_{n}(u) \geqslant Z_{n \mid E-u}^{\prime}>0$ for large $n$, by Remark 5.8.] It follows from (6.7) and (6.8) that $Z_{n \mid E}^{\prime}(f)$ is increasing in $E$ and its increments are increasing in $f$. Hence

$$
\begin{aligned}
0 & \leqslant\left[a_{n}(u, g)-a_{n}(s, g)\right] / a_{n}(u) \\
& \leqslant\left[a_{n}(0, h)-a_{n}(s, h)\right] / a_{n}(s)
\end{aligned}
$$

whenever $g \leqslant h$. Let $a>0$ be a constant to be chosen later. We apply the preceding inequality to the two functions

$$
g_{1}=f 1_{\left\{\tilde{N}_{l} \leqslant a\right\}} \text { and } \quad g_{2}=f 1_{\left\{\tilde{N}_{l}>a\right\}}
$$

and the corresponding $h_{1}$ and $h_{2}$ that are obtained by estimating $f$ according to (2.11). After a spatial averaging we arrive at the estimate

$$
\begin{aligned}
0 & \leqslant \bar{M}_{n}^{\prime \prime}(f)-r_{n} \bar{M}_{n}^{s}(f) \\
& \leqslant c(1+a)\left(q_{n}-1\right)+c \tilde{q}_{n} \bar{M}_{n \mid E+s}^{s}\left(\tilde{N}_{l} ; \tilde{N}_{l}>a\right)
\end{aligned}
$$

Here $1 \geqslant r_{n} \equiv a_{n}(s) / a_{n}(u) \geqslant Z_{n \mid E-s}^{\prime} / Z_{n \mid E}^{\prime} \rightarrow 1$ as $n \rightarrow \infty$ by Lemma 6.4, and similarly

$$
1 \leqslant q_{n} \equiv a_{n}(0) / a_{n}(s) \leqslant Z_{n \mid E}^{\prime} / Z_{n \mid E-s}^{\prime} \rightarrow 1
$$

and $\tilde{q}_{n} \equiv Z_{n \mid E+s}^{\prime} / Z_{n \mid E-s}^{\prime} \rightarrow 1$ as $n \rightarrow \infty$. By Lemma 6.2,

$$
\lim \sup \bar{M}_{n \mid E+s}^{s}\left(\tilde{N}_{l} ; \tilde{N}_{1}>a\right)=\lim \sup \tilde{M}_{n \mid E+s}^{s}\left(\tilde{N}_{l} ; \tilde{N}_{1}>a\right)
$$


and (6.5) and Lemma I.5.2 imply that the last expression tends to zero as $a \rightarrow \infty$. Combining these estimates, we arrive at the desired conclusion. In the case $b c=$ per we simply omit the spatial averaging in the preceding argument.

Rest of the Proof of Theorem 3.3. It is sufficient to consider suitable subsubsequences of arbitrary subsequences. We can therefore assume that $\min (u, 1)$ converges to a limit $q$. If $q>0$, we are in the case (6.2), which was already treated. In the alternative case, we can choose a sequence $(s)$ as in the hypothesis of Proposition 6.6, and the validity of the theorem for the sequences $\left(\bar{M}_{n \mid \mathrm{bc}}^{s}\right)$ and $\left(M_{n \mid \mathrm{per}}^{s}\right)$ carries over to the sequences $\left(\bar{M}_{n \mid \mathrm{bc}}^{u}\right)$ and $\left(M_{n \mid \mathrm{per}}^{u}\right)$.

\section{THE VARIATIONAL PRINCIPLE FOR GIBBS MEASURES}

This final section contains the proof of Theorem 3.4. To obtain a convenient expression for the excess mean free energy we first need the grand canonical analog of Theorem 3.2, namely the existence and variational characterization of the pressure.

Proposition 7.1. Let $z, \beta>0$ and $p(z, \beta)$ be defined by (3.11). Then

$$
\lim _{n \rightarrow \infty} v_{n}^{-1} \log \Xi_{n, z, \beta, \mathrm{bc}}=p(z, \beta)
$$

for each bc $\in \Omega \cup\{$ per $\}$, and the convergence is uniform in bc $\in \Omega(t) \cup\{$ per $\}$ for each $t>0$.

Proof. In the pure positional setting of particles without momentum, this result was established in ref. 2 . The present case can be reduced to the positional one as follows. For $P \in \mathscr{P}_{\Theta}$ we let $\hat{P}=P(\omega: \hat{\omega} \in \cdot)$ denote the distribution of the configuration of particle positions in $\hat{\Omega}$, the set of all locally finite subsets $\hat{\omega}$ of $\mathrm{R}^{d}$. In particular, if $Q^{z \beta}$ is the Poisson point random field on $\mathbf{R}^{d} \times \mathrm{R}^{d}$ with intensity measure $\mu(d x, d p)=z d x e^{-\beta|p|^{2}} d p$ [which amounts to setting $\tau(p)=-\log z+\beta|p|^{2}$ in (4.1)], then $Q^{z, \beta}$ is the Poisson point random field on $\mathrm{R}^{d}$ with particle density $c(z, \beta)=z c(\beta)$, and we can write

$$
\Xi_{n, z, \beta, \mathrm{bc}}=\exp \left[v_{n} c(z, \beta)\right] \hat{Q}_{n}^{z-\beta}\left(\exp \left[-\beta H_{n, \mathrm{bc}}^{\mathrm{pot}}\right]\right)
$$

because $H_{n, \text { bc }}^{\text {pot }}(\omega)$ is a function of $\hat{\omega}$. Theorems II.2 and II.3 imply that

$$
\lim _{n \rightarrow \infty} v_{n}^{-1} \log \hat{Q}_{n}^{z, \beta}\left(\exp \left[-\beta H_{n, \mathrm{bc}}^{\mathrm{pot}}\right]\right)=-\min _{P \in \mathscr{P}_{\theta}}\left[\beta U^{\mathrm{pot}}(\hat{P})+\hat{I}_{z, \beta}(\hat{P})\right]
$$


where $\hat{I}_{z, \beta}(\hat{P})=\lim _{n \rightarrow \infty} v_{n}^{-1} I\left(\hat{P}_{n} ; \hat{Q}_{n}^{z, \beta}\right)$ is defined in analogy to Lemma 4.1. On the other hand, (3.10) and (4.2) show that

$$
p(z, \beta)=c(z, \beta)-\min _{P \in \mathscr{P}_{\theta}}\left[\beta U^{\mathrm{pot}}(P)+I_{z, \beta}(P)\right]
$$

where $I_{z, \beta}$ is the mean relative entropy with respect to $Q^{z, \beta}$. We thus only need to show that the minima on the right-hand sides of (7.1) and (7.2) coincide. This will follow one we have shown that, for each $P \in \mathscr{P}_{\theta}$, $\hat{I}_{z, \beta}(\hat{P}) \leqslant I_{z, \beta}(P)$ with equality when $P$ is Maxwellian, in that

$$
P=\int \hat{P}(d \hat{\omega}) \Gamma_{\beta}(\hat{\omega}, \cdot)
$$

Here $\Gamma_{\beta}(\hat{\omega}, \cdot)$ is the conditional distribution of $Q^{z, \beta}$ given $\hat{\omega}$, i.e., the distribution of $\left\{\left(x, Y_{x}\right): x \in \hat{\omega}\right\}$, where the $Y_{x}$ are i.i.d. centered normal with variance $1 / 2 \beta$.

To verify this inequality we can assume that $I_{z, \beta}(P)<\infty$. Then, for all $n, \quad P_{n} \ll Q_{n}^{z . \beta}$ with a density $f_{n}$ whence $\hat{P}_{n} \ll \hat{Q}_{n}^{z . \beta}$ with density $\hat{f}_{n}(\hat{\omega})=\Gamma_{\beta}\left(\hat{\omega}, f_{n}\right)$. Thus, by Jensen's inequality,

$$
\begin{aligned}
I\left(\hat{P}_{n} ; \hat{Q}_{n}^{z, \beta}\right) & =\hat{Q}_{n}^{z, \beta}\left(\hat{f}_{n} \log \hat{f}_{n}\right) \\
& \leqslant \int \hat{Q}_{n}^{z, \beta}(d \hat{\omega}) \Gamma_{\beta}\left(\hat{\omega}, f_{n} \log f_{n}\right)=I\left(P_{n} ; Q_{n}^{z, \beta}\right)
\end{aligned}
$$

with equality when $f_{n}(\omega)$ only depends on $\hat{\omega}$. This gives the desired relationship between $\hat{I}_{z, \beta}$ and $I_{z, \beta}$.

The preceding proposition shows that the excess mean free energy can be characterized as a mean relative entropy relative to the grand canonical Gibbs distributions with free boundary condition.

Corollary 7.2. For all $z, \beta>0$ and $P \in \mathscr{P}_{\boldsymbol{\theta}}$,

$$
\delta F_{z, \beta}(P) \equiv F_{z, \beta}(P)+p(z, \beta)=\lim _{n \rightarrow \infty} v_{n}^{-1} I\left(P_{n} ; G_{n, z, \beta, \text { ree }}\right)
$$

Proof. Since $G_{n, z, \beta \text {, free }}$ is equivalent to $L_{n}$ with the density appearing in (2.17), we have

$$
I\left(P_{n} ; G_{n, z, \beta, \text { free }}\right)=-S_{n}(P)-P\left(N_{n}\right) \log z+\beta P\left(H_{n}\right)+\log \Xi_{n, z, \beta, \text { free }}
$$

for all $n$, so that the corollary follows from Proposition 3.1, Eqs. (3.1) and (3.10), and Proposition 7.1. 
We now turn to the proof of Theorem 3.4. We can assume without loss that $z=\beta=1$ (because this only amounts to a rescaling of the position and momentum spaces together with a rescaling of $\varphi$ ), and we shall drop all indices referring to these parameters. In particular, we write $G_{n}$, resp. $G_{n, \zeta}$, for the grand canonical Gibbs distribution in $A_{n}$ with free boundary condition, resp. $b c=\zeta$. We shall also need the Gibbs distributions in more general sets $\Lambda$ in place of $\Lambda_{n}$, which will be denoted by $G_{A}$, resp. $G_{A, \zeta}$. The sets $A$ and $\Delta$ considered below will always be finite unions of the unit cells $C+i, i \in \mathrm{Z}^{d}$.

Our first aim is the proof of that part of the variational principle which is essential for the equivalence of ensembles, namely that all minimizers of the free energy are Gibbsian. For this we shall need an estimate of the expected variational distance

$$
\int P(d \zeta)\left\|G_{0,5}-G_{0,5 k}\right\|
$$

(relative to certain measures $P$ ) between the Gibbs distributions in $A_{0}=C$ with boundary conditions $\zeta$ and $\zeta_{k}=\zeta \cap\left(A_{k} \times \mathbf{R}^{d}\right)$. Clearly, if $\varphi$ has finite range, then this distance vanishes as soon as $k$ exceeds the range of $\varphi$. The general case will be treated in Lemma 7.4; the next lemma serves as preparation.

For each $0 \neq i \in Z^{d}$ we write $\psi_{i}=\psi(d(C, C+i))$, where $\psi$ is as in assumption (Al) and $d(C, C+i)$ is the distance of the cells $C$ and $C+i$. We set $\psi_{0}=0$. We also set $\Psi=\sum_{i \in Z^{d}} \psi_{i}$ and $\tilde{\psi}_{i}=\psi_{i} / 2 \Psi$, so that $\sum_{i \in Z^{d}} \tilde{\psi}_{i}=1 / 2$.

Lemma 7.3. For every exponent $\gamma \geqslant 1$ there exists a constant $a_{\gamma}<\infty$ such that

$$
G_{0, \zeta}\left(N_{0}^{\gamma}\right) \leqslant a_{\gamma^{\prime}}+\sum_{i \in Z^{d}} \tilde{\psi}_{i} N_{C+i}(\zeta)^{\gamma}
$$

for all $\zeta \in \Omega^{*}$.

Proof. This is a version of Lemma 2 of Dobrushin. ${ }^{(25)}$ For completeness we indicate the proof. Let $b_{\zeta}=\sum_{i \in Z^{d}} \widetilde{\psi}_{i} N_{C+i}(\zeta)$. it is easy to see that $b_{\zeta}<\infty$ for $\zeta \in \Omega^{*}$ (cf. Lemma II.4.2), and we only need to show that

$$
G_{0, \zeta}\left(N_{0}^{\gamma} ; N_{0}>b_{\zeta}\right) \leqslant a_{\gamma}
$$

In view of (5.3) and (2.1),

$$
H_{0, \zeta}^{\text {pot }} \geqslant h\left(N_{0}\right)-b^{\prime} N_{0}-2 \Psi N_{0} b_{\zeta}
$$


On the other hand, $\Xi_{0, \zeta} \geqslant 1$. The left-hand side of $(7.3)$ is therefore at most

$$
\sum_{l>b_{\zeta}} l^{\prime} \exp \left[-h(l)+b^{\prime} l+2 \Psi l^{2}\right] c(1)^{l} / l !
$$

and the corresponding sum over all $l \geqslant 0$ is finite because $h(l) / l^{2} \rightarrow \infty$ as $l \rightarrow \infty$.

Lemma 7.4. Let $P \in \mathscr{P}_{\Theta}$ be such that $t_{P} \equiv P\left(N_{C}^{2}\right)<\infty$. Then for any $\delta>0$ we can find a number $k_{0}$ such that for all $k \geqslant k_{0}$

$$
\int P(d \zeta)\left\|G_{0, \zeta}-G_{0, \zeta k}\right\|<\delta
$$

and, for each $\Lambda$, the same inequality holds when $P$ is replaced by

$$
P G_{A} \equiv \int P(d \eta) G_{A, \eta}\left(\zeta: \zeta \cup\left(\eta \backslash A \times \mathrm{R}^{d}\right) \in \cdot\right)
$$

Proof. A standard estimate [see ref. 24, p. 33, proof of (b)] and a symmetry argument yield for each $\zeta \in \Omega^{*}$

$$
\left\|G_{0, \zeta}-G_{0, \zeta_{k}}\right\| \leqslant 2 L_{0}\left(\left|\exp \left[-H_{0, \zeta}\right]-\exp \left[-H_{0, \zeta_{k}}\right]\right|\right) / \max \left(\Xi_{0, \zeta}, \Xi_{0, \zeta_{k}}\right)
$$

Decomposing the integral above into parts according to the sign of $H_{0, \zeta}-H_{0, \zeta k}$ and using (2.1), we obtain the upper bound

$$
2\left[G_{0 . \zeta}\left(N_{0}\right)+G_{0 . \zeta \zeta_{k}}\left(N_{0}\right)\right] \sum_{i \in Z^{d} \backslash A_{k}} \psi_{i} N_{C+i}(\zeta)
$$

when $k \geqslant r(\varphi)$. Applying Lemma 7.3 for $\gamma=1$, integrating over $\zeta$, and using the Cauchy-Schwarz inequality, we find

$$
\int P(d \zeta)\left\|G_{0 . \zeta}-G_{0 . \zeta_{k}}\right\| \leqslant\left(4 a_{1} t_{P}^{1 / 2}+2 t_{P}\right) \delta_{k}
$$

where $\delta_{k} \equiv \sum_{i \in Z^{d} \backslash A_{k}} \psi_{i}$ tends to zero as $k \rightarrow \infty$. This proves the first assertion.

The same kind of estimate also gives the second assertion, provided we can find a constant $t<\infty$ such that $P G_{A}\left(N_{C+i}^{2}\right) \leqslant t$ for all $\Lambda$ and all $i \in Z^{d}$. To show this, we use again an idea of Dobrushin. ${ }^{(25)}$ Since $P G_{A}\left(N_{C+i}^{2}\right)=t_{P}$ when $i \in Z^{d} \backslash \Lambda$ we only need to consider the case when $i \in Z^{d} \cap A$. We define

$$
t_{A}=\max _{i \in Z^{d} \cap A} P G_{A}\left(N_{C+i}^{2}\right)
$$


In view of the consistency of the Gibbs distributions and by Lemma 7.3 we have for each $i \in Z^{d} \cap A$

$$
\begin{aligned}
P G_{A}\left(N_{C+i}^{2}\right) & =\int P G_{A}(d \zeta) G_{C+i, \zeta}\left(N_{C+i}^{2}\right) \\
& \leqslant a_{2}+\sum_{j \in Z^{d}} \tilde{\psi}_{i-j} P G_{A}\left(N_{C+j}^{2}\right)
\end{aligned}
$$

and therefore $t_{A} \leqslant a_{2}+\frac{1}{2} \max \left(t_{A}, t_{P}\right)$. On the other hand, $t_{A}<\infty$ because

$$
t_{\Lambda} \leqslant \int P(d \zeta) G_{A, \zeta}\left(N_{A}^{2}\right) \leqslant a^{\prime}+\sum_{i \in Z^{d} \backslash A} \psi(d(C+i, \Lambda)) P\left(N_{C}^{2}\right)
$$

for some $a^{\prime}=a^{\prime}(\Lambda)<\infty$ by an obvious variant of Lemma 7.3. It follows that $t_{A} \leqslant t \equiv \max \left(t_{P}, 2 a_{2}\right)$, and the proof is complete.

After these preparations we can enter into the proof that the minimizers of the mean free energy are tempered Gibbs measures. We follow a well-known idea of Preston. ${ }^{(23,24)}$ let $P \in \mathscr{P}_{\Theta}$ be such that $\delta F(P)=0$. Then $U^{\text {pot }}(P)<\infty$ and thus $t_{P}=P\left(N_{C}^{2}\right)<\infty$. Hence Lemma 7.4 is applicable. Moreover, the ergodic theorem [for the discrete translation group $\left(\vartheta_{i}\right)_{i \in Z^{d}}$ ] shows that $P\left(\Omega^{*}\right)=1$, i.e., $P$ is tempered. On the other hand, we conclude from Corollary 7.3 that $P_{n} \ll G_{n}$ when $n$ is large enough. By translation invariance this means that, for all sufficiently large cubes $A, P_{A}$ is absolutely continuous with respect to $G_{A}$ with a density $g_{A}$. Here $P_{A}$ is the restriction of $P$ to the events in $A$, i.e., the image of $P$ under the mapping $\omega \rightarrow \omega_{\Lambda}=\omega \cap\left(\Lambda \times \mathrm{R}^{d}\right)$, and $G_{A}$ is the Gibbs distribution in $\Lambda$ with free boundary condition. In particular, for any $\Delta \subset \Lambda$ we consider the restriction $G_{A, \Delta}=\left(G_{A}\right)_{\Delta}$ of $G_{A}$ to the events in $\Delta$, and we have that $P_{\Delta} \ll G_{A . \Delta}$ with density

$$
g_{A, A}(\zeta)=\int G_{A \backslash \Delta, \zeta_{A}}(d \omega) g_{A}\left(\omega \cup \zeta_{\Delta}\right)
$$

The crucial consequence of the assumption $\delta F(P)=0$ is the following.

Lemma 7.5. Let $P \in \mathscr{P}_{\theta}$ be such that $\delta F(P)=0$, and let $k \geqslant 1$ and $\delta>0$ be given. Then there exist two sets $\Delta, A \subset \mathrm{R}^{d}$ such that $\Lambda_{k} \subset \Delta \subset A$ and

$$
G_{A}\left(\left|g_{A, \Delta}-g_{A, \Delta \backslash C}\right|\right)<\delta
$$


Proof. In view of the argument in ref. 24, p. 324, Step 2, it is sufficient to show that for any $\delta>0$ we can find $\Delta$ and $A$ such that $\Lambda_{k} \subset \Delta \subset A$ and

$$
I_{A}\left(P ; G_{A}\right)-I_{\Delta \backslash C}\left(P ; G_{A}\right)<\delta
$$

Here we write $I_{\Delta}(\cdot ; \cdot)$ for the relative entropy of measures that are restricted to the events in $\Delta$. Using Corollary 7.2 , we see that there exists some $n \geqslant k$ such that $v_{n}^{-1} I\left(P_{n} ; G_{n}\right) \leqslant \delta / 2^{d} v_{k}$. As in ref. 24, p. 324, Step 1, we choose an $m$ with $m^{d} v_{k} \leqslant v_{n} \leqslant(2 m)^{d} v_{k}$ and disjoint translates $\Lambda_{k}(l)=$ $A+i(l) \subset \Lambda_{n}, i(l) \in Z^{d}, 1 \leqslant l \leqslant m^{d}$. Writing $\Delta(l)=\Lambda_{k}(1) \cup \cdots \cup \Lambda_{k}(l)$, we find

$$
\begin{aligned}
& m^{-d} \sum_{l=1}^{m^{d}}\left[I_{\Delta(l)}\left(P ; G_{n}\right)-I_{\Delta(l) \backslash(C+i(l))}\left(P ; G_{n}\right)\right] \\
& \quad \leqslant m^{-d} I_{\Delta\left(m^{d}\right)}\left(P ; G_{n}\right)<\delta
\end{aligned}
$$

So for at least one $l$ the corresponding term in the sum above is less than $\delta$, and by translation invariance we obtain (7.4) for $A=\Lambda_{n}-i(l)$ and $\Delta=\Delta(l)-i(l)$.

We are now ready for the first direction of the variational principle.

Proposition 7.6. Each $P \in \mathscr{P}_{\Theta}$ with $\delta F(P)=0$ is a tempered Gibbs measure.

Proof. We saw already that $P$ is tempered and $t_{P}<\infty$. We need to establish Eq. (2.18) for any measurable $f \geqslant 0$. We can clearly assume that $f \in \mathscr{L}$ and $0 \leqslant f \leqslant 1$. For given $\delta>0$ we let $k$ be so large that $f$ only depends on the particles in $A_{k}$ and the conclusions of Lemma 7.4 hold, and we determine $\Delta$ and $\Lambda$ according to Lemma 7.5 .

Consider the term on the right-hand side of Eq. (2.18) for $n=0$. We replace its inner integral

$$
G_{0} f(\zeta) \equiv \int G_{0, \zeta}(d \omega) f\left(\omega_{0} \cup \zeta \backslash \zeta_{0}\right)
$$

by $\tilde{f}_{k}(\zeta) \equiv G_{0} f\left(\zeta_{k}\right)$. Since $f$ only depends on the particles in $\Lambda_{k}$, this replacement leads to an error of at most $\int P(d \zeta)\left\|G_{0, \zeta}-G_{0, \zeta_{k}}\right\|<\delta$. Since $\widetilde{f}_{k}$ only depends on the particles in $A_{k} \backslash C \subset \Delta \backslash C$ and $P \ll G_{A}$ on the events which occur in this set,

$$
P\left(\tilde{f}_{k}\right)=G_{\Lambda}\left(g_{\Lambda, \Delta \backslash C} \tilde{f}_{k}\right)
$$


Changing $\widetilde{f}_{k}$ in the last term back into $G_{0} f$ gives an error of at most

$$
\begin{aligned}
& \int G_{A}(d \zeta) g_{A, \Delta \backslash C}(\zeta)\left\|G_{0, \zeta}-G_{0, \zeta k}\right\| \\
& \quad=\int P G_{A \backslash \Delta \backslash C)}(d \zeta)\left\|G_{0, \zeta}-G_{0, \zeta k}\right\|<\delta
\end{aligned}
$$

(For the equality above we first use the fact that $G_{A \backslash \Delta \backslash C), \omega_{\Delta \backslash C}}$ is the conditional distribution of $G_{A}$ given the configuration $\omega_{\Delta \backslash C}$ in $\Delta \backslash C$, and then that $P$ has density $g_{A, \Delta \backslash C}$ relative to $G_{A}$ on events occurring in $\Delta \backslash C$.) As a next step we observe that

$$
G_{A}\left(g_{A, \Delta \backslash C} G_{0} f\right)=G_{A}\left(g_{A, \Delta \backslash C} f\right)
$$

because $g_{A, \Delta \backslash C}$ does not depend on the particles in $A_{0}=C$ and $G_{0, \zeta}$ is the suitable conditional distribution of $G_{A}$. According to the choice of $\Delta$ and $\Lambda$, we may finally replace $g_{A, \Delta \backslash C}$ by $g_{A, \Delta}$ with an error less than $\delta$. In this way we arrive at the term $G_{A}\left(g_{A, \Delta} f\right)=P(f)$. We have thus shown that

$$
\left|P\left(G_{0} f\right)-P(f)\right|<3 \delta
$$

and this gives (2.18) for $n=0$. To obtain (2.18) for general $n$ we can either apply Theorem (1.33) of ref. 24 or simply repeat the preceding argument on a new spatial scale.

We finally come to the converse part of the variational principle.

Proposition 7.7. $\delta F$ vanishes on the set of tempered Gibbs measures.

Proof. We might deduce this result from the large-deviation principle for tempered Gibbs measures in ref. 2 by exploiting the fact that a good rate function in a large deviation principle vanishes at the underlying measure whenever this is ergodic. But we prefer a more direct argument which requires only the somewhat weaker Proposition 7.1.

Let $P$ be a tempered Gibbs measure. We need that $t_{P}=P\left(N_{C}^{2}\right)<\infty$. This follows either from subtle direct analysis, namely the superstability estimates of Ruelle, ${ }^{(20)}$ or from general theory by noting that (by the ergodic decomposition of translation invariant tempered Gibbs measures ${ }^{(24)}$ and the fact that $\delta F$ is measure affine) $P$ can be assumed to be ergodic; in which case $t_{P}<\infty$ follows from the temperedness by means of the ergodic theorem. 
We need to show that the limit in Corollary 7.2 vanishes. We fix any $n \geqslant 0$. By (2.17) and (2.18), $P_{n} \ll G_{n}$ with density

$$
\begin{aligned}
g_{n}(\omega) & =\int P(d \zeta) d G_{n, \zeta} / d G_{n}(\omega) \\
& =\int P(d \zeta) \exp \left[H_{n}(\omega)-H_{n, \zeta}(\omega)\right] \Xi_{n} / \Xi_{n, \zeta}
\end{aligned}
$$

Therefore we can write, using Jensen's inequality, (2.18), and the lower regularity of $\varphi$,

$$
\begin{aligned}
I\left(P_{n} ; G_{n}\right) & =G_{n}\left(g_{n} \log g_{n}\right) \\
& \leqslant G_{n}\left(\int P(d \zeta)\left(d G_{n, \zeta} / d G_{n}\right) \log d G_{n, \zeta} / d G_{n}\right) \\
& =\int P(d \omega)\left[H_{n}(\omega)-H_{n, \omega}(\omega)+\log \Xi_{n}-\log \Xi_{n, \omega}\right] \\
& \leqslant t_{P} \sum_{i \in Z^{d} \cap A_{n}, j \in Z^{d} \backslash A_{n}} \psi_{i-j}+\log \Xi_{n}-\int P(d \zeta) \log \Xi_{n, \zeta}
\end{aligned}
$$

After division by $v_{n}$, the first of the last three terms converges to 0 , and Proposition 7.1 ensures that the second term converges to $p(1,1)$. On the other hand we have $\Xi_{n, \zeta} \geqslant 1$, so that the same proposition together with Fatou's lemma and the temperedness of $P$ implies that

$$
\liminf _{n \rightarrow \infty} v_{n}^{-1} \int P(d \zeta) \log \Xi_{n, \zeta} \geqslant p(1,1)
$$

It follows that $\delta F(P)=0$, and the proof is complete.

\section{REFERENCES}

1. H. O. Georgii and H. Zessin, Large deviations and the maximum entropy principle for marked point random fields, Prob. Theory Related Fields 96:177-204 (1993).

2. H. O. Georgii, Large deviations and the equivalence of ensembles for Gibbsian particle systems with superstable interaction, Prob. Theory Related Fields 99:171-195 (1994).

3. D. Ruelle, Statistical Mechanics. Rigorous Results (Benjamin, New York, 1969).

4. O. E. Lanford, Entropy and equilibrium states in classical statistical mechanics, in Statistical Mechanics and Mathematical Problems, A. Lenard, ed. (Springer, Berlin, 1973).

5. A. Martin-Löf, Statistical Mechanics and the Foundations of Thermodynamics (Springer, Berlin, 1979).

6. A. E. Khinchin, Mathematical Foundations of Statistical Mechanics (Dover, New York, 1949). 
7. A. M. Halfina, The limiting equivalence of the canonical and grand canonical ensembles (low density case), Math. USSR Sbornik 9:1-52 (1969).

8. R. A. Minlos and A. Haitov, Limiting equivalence of thermodynamic ensembles in case of one-dimensional systems, Trans. Moscow Math. Soc. 32:143-180 (1975).

9. R. L. Dobrushin and B. Tirozzi. The central limit theorem and the problem of equivalence of ensembles, Commun. Math. Phys. 54:173-192 (1977).

10. M. Campanino, G. Del Grosso, and B. Tirozzi, Local limit theorem for Gibbs random fields of particles and unbounded spins, J. Math. Phys. 20:1752-1758 (1979).

11. A. Martin-Löf, The equivalence of ensembles and the Gibbs phase rule for classical lattice systems, J. Stat. Phys. 20:557-569 (1979).

12. R. L. Thompson, Equilibrium states on thin energy shells, Mem. Am. Math. Soc. 1974:150 (1974).

13. H. O. Georgii, Canonical Gibbs Measures (Springer, Berlin, 1979)

14. M. Aizenman, S. Goldstein, and J. L. Lebowitz, Conditional equilibrium and the equivalence of microcanonical and and grandcanonical ensembles in the thermodynamic limit, Common. Math. Phys. 62:279-302 (1978).

15. C. Preston, Canonical and microcanonical Gibbs states, Z. Wahrsch. Verw. Gebiete 40:125-158 (1979).

16. M. Pirlot, Generalized canonical states, Ann. Sci. Univ. Clermont-Ferrand II. Prob. Appl. 4:69-91 (1985).

17. P. Vanheuverzwijn, Discrete lattice systems and the equivalence of microcanonical, canonical and grand canonical Gibbs states, Commun. Math. Phys. 101:153-172 (1985).

18. K. Matthes, J. Kerstan, and J. Mecke, Infinitely Divisible Point Processes (Wiley, Chichester, 1978).

19. T. Tjur, Probability Based on Radon Measures (Wiley, Chichester, 1980).

20. D. Ruelle, Superstable interactions in classical statistical mechanics, Commun. Math. Phys. 18:127-159 (1970).

21. R. Rechtmann and O. Penrose, Continuity of the temperature and derivation of the Gibbs canonical distribution in classical statistical mechanics, J. Stat. Phys. 19:359-366 (1978).

22. O. E. Lanford and D. Ruelle, Observables at infinity and states with short range correlations in statistical mechanics, Commun. Math. Phys. 9:327-338 (1968).

23. C. Preston, Random Fields (Springer, Berlin, 1976).

24. H. O. Georgii, Gibbs Measures and Phase Transitions (de Gruyter, Berlin, 1988).

25. R. L. Dobrushin, Gibbsian random fields for particles without hard core, Theor. Math. Phys. 4:705-719 (1970).

26. H. O. Georgii, Large deviations and maximum entropy principle for interacting random fields on $\mathbb{Z}^{d}$, Ann. Prob. 21:1845-1875 (1993). 